\title{
Inherent and Apparent Scattering Properties of Coated or Uncoated Spheres \\ Embedded in an Absorbing Host Medium
}

\author{
P. Yang, B.-C. Gao, W. J. Wiscombe, M.I. Mishchenko, S. Platnick, H.-L. Huang, \\ B.A.Baum, Y.X. Hu, D. Winker, S.-C. Tsay, S.K. Park
}

\begin{abstract}
The conventional Lorenz-Mie formalism is extended to the scattering process associated with a coated sphere embedded in an absorbing medium. It is shown that apparent and inherent scattering cross sections of a scattering particle, which are identical in the case of transparent host medium, are different if the host medium is absorptive. Here the inherent single-scattering properties are derived from the near-field information whereas the corresponding apparent counterparts are derived from the far-field asymptotic form of the scattered wave with scaling of host absorption that is assumed to be in an exponential form. The formality extinction and scattering efficiencies defined in the same manner as in the conventional sense can be unbounded. For a nonabsorptive particle embedded in an absorbing medium, the effect of host absorption on the phase matrix elements associated with polarization is significant. This effect, however, is largely reduced for strongly absorptive particles such as soot. For soot particles coated with water, the impurity can substantially reduce the single-scattering albedo of the particle if the size parameter is small. For water-coating soot and hollow ice spheres, it is shown that the phase matrix elements $-P_{12} / P_{11}$ and $P_{33} / P_{11}$ are unique if the shell is thin, as compared with the case for thick shell. Furthermore, the radiative transfer
\end{abstract}


equation regarding a multidisperse particle system in an absorbing medium is discussed. It is illustrated that the conventional computational algorithms can be applied to solve the multiple scattering process if the scaled apparent single-scattering properties are applied.

OCIS codes: $010.1290,010.1310,010.3920,290.5850,290.1090,280.1310$

P. Yang, W. J. Wiscombe, S Plantick, S.-C. Tsay, S.K. Park are with Code 913, NASA Goddard Space Fight Center, Greenbelt, MD 20771 (P. Yang is also with Goddard Earth Science and Technology Center, University of Maryland Baltimore County). B.-C. Gao is with the Remote Sensing Division, code 7212, U.S. Naval Research Laboratory, Washington, D.C. 20375. M. I. Mishchenko is with NASA Goddard Institute for Space Studies, 2880 Broadway, New York, NY 10025. H.-L. Huang is with Cooperative Institute for Meteorological Satellite Studies, University of Wisconsin-Madison, 1225 West Dayton Street, Madison, WI 53706. B.A. Baum, Y.X. Hu, and D. M. Winker are with Atmospheric Sciences Division, MS 420, NASA Langley Research Center, Hampton, VA 23681. S.K. Park is also with Science Systems and Applications, Inc., 5900 Princess Garden Pkwy, Suite 300, Lanham, MD 20706. The email address for P. Yang is pyang@climate.gsfc.nasa.gov 


\section{Introduction}

The geometrical shapes of many micron-sized natural particles including cloud droplets can be well approximated as spheres in light scattering calculations. The LorenzMie theory ${ }^{1}$ provides the theoretical basis for the interaction between a sphere and an electromagnetic wave (e.g., Ref. 2-5). Numerically stable and efficient algorithms and the corresponding computational codes have been developed ${ }^{4-8}$ for deriving the exact single scattering properties of spherical particles. Although the scattering problem associated with spheres, involved in many applications including aircraft and satellite remote sensing, seems to be solved, an issue on this subject still needs to be addressed. In the conventional Lorenz-Mie formulation, the host medium within which the sphere is embedded is assumed to be a nonabsorptive dielectric material such as air. For many situations, the medium surrounding the spherical particles may contain constituents with significant absorption. For example, ozone and $\mathrm{CO}_{2}$ in the atmosphere have strong absorptive bands at 9.6 and $15 \mu \mathrm{m}$, respectively. Additionally, atmospheric water vapor provides a strongly absorbing component to the air surrounding spherical aerosol or water particles in both solar and infrared spectral regions.

For scattering of solar and infrared radiation by atmospheric particles embedded in an absorbing medium, the absorption effect of the host medium on the scattering properties of the particles may not be negligible. When the host medium is transparent (i.e., a dielectric medium with a purely real refractive index), the use of the standard Lorenz-Mie formulation does not raise any difficulty. In this case, the effect of the host medium can be accounted for by determining a relative refractive index for the scattering particle, which is defined as the ratio of the particle refractive index to that of its host 
counterpart. Unfortunately, the scattering properties of a sphere in an absorbing medium cannot be obtained by simply modifying the refractive indices for the particle and the host medium along with scaling the incident wavelength in the input list of the conventional Mie computational code. Thus, there is a necessity of reformulating the conventional Lorenz-Mie theory to accommodate a spherical particle embedded in an absorbing medium.

The scattering properties of a homogenous sphere in an absorbing medium have been investigated by several authors ${ }^{9-12}$. On this specific issue, two approaches that are based either upon the asymptotic form of electromagnetic field in the radiation zone (i.e., the far-field) ${ }^{9}$ or upon the information of the field at particle surface (i.e., the nearfield $)^{11,12}$ have been used in the previous calculation of the extinction and scattering cross sections. When the host medium is absorptive, the host absorption has not only attenuated the scattered wave in magnitude but also modulated the wave mode when the wave reaches the radiation zone. Thus, for an observation in the radiation zone, the particle's inherent optical properties are coupled with the absorption effect of the medium in an inseparable manner. For this reason, the scattering properties of the particle that are derived from the far-field asymptotic form of the scattered wave, though rescaled by removing the exponential absorption of the host medium between the particle and the observational point, are the apparent optical properties of the scattering particle. The apparent scattering and extinction cross sections for a sphere in an absorbing medium have been derived by Mundy et al. ${ }^{9}$, which unfortunately may be implausible at times because the corresponding extinction efficiency can be smaller than the scattering efficiency. This discrepancy is caused by neglecting the absorption of the incident wave 
within the host medium in the calculation of the extinction cross section, as is shown by Chylek ${ }^{10}$. The true or inherent scattering and absorption cross sections of the particle can be calculated by integrating the Poynting vector at the scattering particle's surface as shown by Chylek ${ }^{10}$. The apparent optical properties reduce to their corresponding inherent counterparts if the absorption of the host medium is absent. Most recently, Sudiarta and Chylek" reported the numerical calculations for the inherent extinction and scattering efficiencies based on the theoretical frame developed in Chylek's previous work $^{10}$. Similar calculations were reported by $\mathrm{Fu}$ and $\mathrm{Sun}^{12}$ who also investigated the effect of absorption within the host medium on the phase function and asymmetry factor.

The inherent extinction and scattering efficiencies have limited applications in practice for three reasons. First, these efficiencies do not have the conventional meanings in that the corresponding cross sections are not simply the products of these efficiencies and the geometrical projected-area of the particle. In fact, since the incident irradiance spatially varies when the host medium is absorptive, a reference plane must be specified to unambiguously define extinction and scattering cross sections. Second, to consider the bulk scattering properties of a polydispersive system, one always deals with the far field and consequently, the apparent optical properties. Third, the motivation to determine particle single scattering properties is primarily for radiative transfer calculations that require both cross sections and an accurate description of the phase matrix. It is not selfconsistent to use the inherent cross sections in radiative transfer calculations when the corresponding phase matrix is an apparent optical property.

The intent of this study is to properly define the apparent extinction and scattering cross sections versus their inherent counterparts. Additionally, we also investigate the 
effect of host absorption on the polarization configuration of the scattered wave since the previous studies focus primarily on the total cross sections and scattered intensity. Furthermore, because the scattering properties of coated spheres such as black carbon aerosols that are coated with water are of interest in many disciplines including atmospheric remote sensing and radiative transfer calculations, we also present a solution for the scattering properties of a coated sphere in an absorbing host medium. The previous solution for a homogeneous sphere in an absorbing medium is a special case of the present study when the core and shell of the coated sphere have the same refractive index. In Section 2 we present the basic mathematical expressions for the inherent and apparent scattering properties of a coated sphere within an absorbing medium. In Section 2 we also discuss the proper form of the single-scattering properties for radiative transfer calculation involving a polydisperse particle system in an absorbing host medium. Presented in Section 3 are the numerical results and discussions. Finally, the conclusions are given in Section 4.

\section{Inherent and Apparent Optical Properties of Coated Spheres in Absorbing}

\section{Medium}

A. Transverse Components of Incident and Scattered Waves in a Spherical Coordinate System for Coated Spheres embedded in Absorbing Medium

In this study we select the time-dependent factor $\exp (-i \omega t)$ for the complex representation of a temporally harmonic electromagnetic wave, where $\omega$ is the angular frequency of the wave. In addition, we employ the Gaussian unit system for the electromagnetic field. Let us consider the scattering of an incident electromagnetic wave 
by a coated sphere embedded in an absorbing medium. As shown in Fig. 1, the complex refractive indices for the particle core, particle shell, and the host medium are $m_{1}, m_{2}$, and $m_{0}$, respectively. Let the incident electric field be polarized along the $\mathrm{x}$-axis and propagate along z-axis of the coordinate system. Thus, the incident electric and magnetic fields can be written as follows:

$$
\begin{aligned}
& \vec{E}_{i}(x, y, z)=\hat{e}_{x} E_{0} \exp \left(i k m_{0} z\right), \\
& \vec{H}_{i}(x, y, z)=-\frac{i}{k} \nabla \times \vec{E}_{i}(x, y, z)=\hat{e}_{y} m_{0} E_{0} \exp \left(i k m_{0} z\right),
\end{aligned}
$$

where $k=\omega / c$ in which $c$ is the speed of light in vacuo, $E_{0}$ is the amplitude of the electric field, and $\hat{e}_{x}$ and $\hat{e}_{y}$ are the unit vectors along the $x$-axis and $y$-axis, respectively. Note that in the present study we assume permeability to be unity. The expansions of incident and scattering fields in terms of spherical harmonics for a coated sphere within an absorbing medium is similar to their counterparts in the case of the conventional Mie formulation. To derive inherent and apparent cross sections and scattering phase matrix, it is sufficient to consider the transverse components of the incident and scattered fields decomposed in a spherical coordinate system. It can be shown that these field components can be expanded in the form

$$
\begin{aligned}
& E_{i \theta}(\phi, \theta, r)=\frac{\cos \phi}{m_{0} k r} \sum_{n=1}^{\infty} E_{n}\left[\pi_{n}(\cos \theta) \psi_{n}\left(m_{0} k r\right)-i \tau_{n}(\cos \theta) \psi^{\prime}{ }_{n}\left(m_{0} k r\right)\right], \\
& E_{i \phi}(\phi, \theta, r)=-\frac{\sin \phi}{m_{0} k r} \sum_{n=1}^{\infty} E_{n}\left[\tau_{n}(\cos \theta) \psi_{n}\left(m_{0} k r\right)-i \pi_{n}(\cos \theta) \psi_{n}^{\prime}\left(m_{0} k r\right)\right], \\
& H_{i \theta}(\phi, \theta, r)=\frac{\sin \phi}{k r} \sum_{n=1}^{\infty} E_{n}\left[\pi_{n}(\cos \theta) \psi_{n}\left(m_{0} k r\right)-i \tau_{n}(\cos \theta) \psi^{\prime}{ }_{n}\left(m_{0} k r\right)\right],
\end{aligned}
$$




$$
\begin{aligned}
& H_{i \phi}(\phi, \theta, r)=\frac{\cos \phi}{k r} \sum_{n=1}^{\infty} E_{n}\left[\tau_{n}(\cos \theta) \psi_{n}\left(m_{0} k r\right)-i \pi_{n}(\cos \theta) \psi_{n}^{\prime}\left(m_{0} k r\right)\right], \\
& E_{s \theta}(\phi, \theta, r)=\frac{\cos \phi}{m_{0} k r} \sum_{n=1}^{\infty} E_{n}\left[i a_{n} \tau_{n}(\cos \theta) \xi_{n}^{\prime}\left(m_{0} k r\right)-b_{n} \pi_{n}(\cos \theta) \xi_{n}\left(m_{0} k r\right)\right], \\
& E_{s \phi}(\phi, \theta, r)=-\frac{\sin \phi}{m_{0} k r} \sum_{n=1}^{\infty} E_{n}\left[i a_{n} \pi_{n}(\cos \theta) \xi_{n}^{\prime}\left(m_{0} k r\right)-b_{n} \tau_{n}(\cos \theta) \xi_{n}\left(m_{0} k r\right)\right], \\
& H_{s \theta}(\phi, \theta, r)=-\frac{\sin \phi}{k r} \sum_{n=1}^{\infty} E_{n}\left[a_{n} \pi_{n}(\cos \theta) \xi_{n}\left(m_{0} k r\right)-i b_{n} \tau_{n}(\cos \theta) \xi_{n}^{\prime}\left(m_{0} k r\right)\right] \\
& H_{s \phi}(\phi, \theta, r)=-\frac{\cos \phi}{k r} \sum_{n=1}^{\infty} E_{n}\left[a_{n} \tau_{n}(\cos \theta) \xi_{n}\left(m_{0} k r\right)-i b_{n} \pi_{n}(\cos \theta) \xi_{n}^{\prime}\left(m_{0} k r\right)\right],
\end{aligned}
$$

where we have employed the common nomenclature for the special functions involved in the Lorenz-Mie algorithm (e.g., Ref. $2,4,5$ ); for example, $\psi_{n}$ and $\xi_{n}$ indicate RiccatiBessel functions associated with spherical Bessel function $j_{n}$ and the Hankel function $h_{n}^{(1)}$, respectively. The functions $\pi_{n}$ and $\tau_{n}$ are the functions of scattering zenith angle and are related to the associated Legendre function $P_{n}^{1}$. The coefficient $E_{n}$ can be determined from the orthogonality of the spherical harmonics and is given by

$$
E_{n}=E_{0} i^{n}(2 n+1) /[n(n+1)] \text {. }
$$

The coefficients $a_{n}$ and $b_{n}$ in Eqs.(2e)-(2h) for the scattered field can be determined from an electromagnetic boundary condition that is imposed at both the interfaces of the core and the shell of the particle and the interface of the scatterer and the host medium. Here, without a detailed derivation, we give these coefficients in a form similar to that presented in Bohren and Huffman ${ }^{5}$ as follows:

$$
a_{n}=\frac{\left[\tilde{D}_{n} m_{0} / m_{2}+n /\left(m_{0} k R_{2}\right)\right] \psi_{n}\left(m_{0} k R_{2}\right)-\psi_{n-1}\left(m_{0} k R_{2}\right)}{\left[\tilde{D}_{n} m_{0} / m_{2}+n /\left(m_{0} k R_{2}\right)\right] \xi_{n}\left(m_{0} k R_{2}\right)-\xi_{n-1}\left(m_{0} k R_{2}\right)},
$$




$$
\begin{gathered}
b_{n}=\frac{\left[\tilde{G}_{n} m_{2} / m_{0}+n /\left(m_{0} k R_{2}\right)\right] \psi_{n}\left(m_{0} k R_{2}\right)-\psi_{n-1}\left(m_{0} k R_{2}\right)}{\left[\tilde{G}_{n} m_{2} / m_{0}+n /\left(m_{0} k R_{2}\right)\right] \xi_{n}\left(m_{0} k R_{2}\right)-\xi_{n-1}\left(m_{0} k R_{2}\right)}, \\
\tilde{D}_{n}=\frac{D_{n}\left(m_{2} k R_{2}\right)-A_{n}\left[\chi_{n}^{\prime}\left(m_{2} k R_{2}\right) / \psi_{n}\left(m_{2} k R_{2}\right)\right]}{1-A_{n}\left[\chi_{n}\left(m_{2} k R_{2}\right) / \psi_{n}\left(m_{2} k R_{2}\right)\right]}, \\
\tilde{G}_{n}=\frac{D_{n}\left(m_{2} k R_{2}\right)-B_{n}\left[\chi_{n}^{\prime}\left(m_{2} k R_{2}\right) / \psi_{n}\left(m_{2} k R_{2}\right)\right]}{1-B_{n}\left[\chi_{n}\left(m_{2} k R_{2}\right) / \psi_{n}\left(m_{2} k R_{2}\right)\right]}, \\
A_{n}=\frac{m_{2} D_{n}\left(m_{1} k R_{1}\right)-m_{1} D_{n}\left(m_{2} k R_{1}\right)}{m_{2} D_{n}\left(m_{1} k R_{1}\right)\left[\chi_{n}\left(m_{2} k R_{1}\right) / \psi_{n}\left(m_{2} k R_{1}\right)\right]-m_{1}\left[\chi_{n}^{\prime}\left(m_{2} k R_{1}\right) / \psi_{n}\left(m_{2} k R_{1}\right)\right]}, \\
B_{n}=\frac{m_{1} D_{n}\left(m_{1} k R_{1}\right)-m_{2} D_{n}\left(m_{2} k R_{1}\right)}{m_{1} D_{n}\left(m_{1} k R_{1}\right)\left[\chi_{n}\left(m_{2} k R_{1}\right) / \psi_{n}\left(m_{2} k R_{1}\right)\right]-m_{2}\left[\chi_{n}^{\prime}\left(m_{2} k R_{1}\right) / \psi_{n}\left(m_{2} k R_{1}\right)\right]},
\end{gathered}
$$

where $R_{1}$ and $R_{2}$ are the radii for the core and the shell of the coated sphere, as is shown in Fig. 1, and $D_{n}$ is the logarithmic derivative of Riccati-Bessel function $\psi_{n}$, that is, $D_{n}(x)=d\left[\ln \psi_{n}(x)\right] / d x$. In the case when the refractive index $m_{0}$ is unity, the coefficients in Eqs. (4a) and (4b) reduce to a form that is exactly identical to that given in Bohren and Huffman ${ }^{5}$. If the sphere is homogeneous, i.e., $m_{2}=m_{1}$, the coefficients, $a_{n}$ and $b_{n}$, reduce to the form

$$
\begin{aligned}
& a_{n}=\frac{\left[m_{0} D_{n}\left(m_{0} k R\right) / m_{1}+n /\left(m_{0} k R\right)\right] \psi_{n}\left(m_{0} k R\right)-\psi_{n-1}\left(m_{0} k R\right)}{\left[m_{0} D_{n}\left(m_{0} k R\right) / m_{1}+n /\left(m_{0} k R\right)\right] \xi_{n}\left(m_{0} k R\right)-\xi_{n-1}\left(m_{0} k R\right)}, \\
& b_{n}=\frac{\left[m_{1} D_{n}\left(m_{1} k R\right) / m_{0}+n /\left(m_{0} k R\right)\right] \psi_{n}\left(m_{0} k R\right)-\psi_{n-1}\left(m_{0} k R\right)}{\left[m_{1} D_{n}\left(m_{1} k R\right) / m_{0}+n /\left(m_{0} k R\right)\right] \xi_{n}\left(m_{0} k R\right)-\xi_{n-1}\left(m_{0} k R\right)},
\end{aligned}
$$

where $\mathrm{R}$ is the radius of the homogeneous sphere. The coefficients in Eqs.(5a) and (5b) are equivalent to the form presented in Kerker $^{3}$ and Ross ${ }^{13}$ that was recaptured in Fu and Sun $^{12}$. Note that the form expressed by Eqs. (5a) and $(5 b)$ is more suitable for numerical computation, as is evident from the extensive discussions in Wiscombe ${ }^{4}$ and Bohren and Huffman $^{5}$. In the numerical computation of Eqs.(4a)-(4f), we have taken the advantage of 
the various numerical techniques suggested by Wiscombe ${ }^{4.7}$, Bohren and Huffman ${ }^{5}$, and Toon and Ackerman ${ }^{8}$. In particular, following Kattawar and Hood $^{14}$, in the computation of the higher-order terms of $a_{n}$ and $b_{n}$ in Eqs. (4a) and (4b) for large size parameters we use the corresponding expressions in the homogeneous case for the two coefficients when $n>\left|m_{1} k R_{\mathrm{l}}\right|$ to preserve numerical significance and also for computational economy.

\section{B. Scattering Phase Matrix and Inherent and Apparent Cross Sections}

For an incident wave propagating in an absorbing medium along the $z$-axis of the coordinate system, the incident irradiance (i.e., the magnitude of Poynting vector) can be written as follows:

$$
F(z)=\left|\frac{c}{8 \pi} \operatorname{Re}\left(\bar{E}_{i} \times \bar{H}_{i}^{*}\right)\right|=F_{0} \exp \left(-2 m_{0, i} k z\right)
$$

where the asterisk indicates complex conjugate, and $m_{0, i}$ is the imaginary part of the refractive index of the host medium. The quantity $F_{0}$ is the incident irradiance at the origin of the coordinate system in the case when the scattering particle does not exist, which, derived on the basis of Eqs. (la) and (lb), equals to $c m_{0, r}\left|E_{0}\right|^{2} /(8 \pi)$ where $m_{0, r}$ is the real part of the refractive index of the host medium. Note that $(c / 8 \pi) \operatorname{Re}\left(\vec{E}_{i} \times \vec{H}_{i}^{*}\right)$ in Eq. (6) is the time-averaged Poynting vector in the Gaussian unit system for a timeharmonic electromagnetic wave. Because the incident irradiance varies with the coordinate value $z$ in the host medium, the definitions of various optical cross sections (i.e., the ratio of corresponding flux to the incident irradiance value at a reference location) are arbitrary in this case. That is, the cross sections depend on the selection of the reference incident irradiance plane. In the present study, we select the reference plane 
through the origin for the incident irradiance because of the advantage of this convention for application to multiple scattering processes as will be shown later. Thus, the interception cross section of the particle for blocking the incident radiation, when it is defined with respect to the incident irradiance at the origin (i.e., the ratio of the incident flux intercepted by the particle to $F_{0}$ ), is then given by

$$
\begin{aligned}
\sigma_{i} & =\frac{1}{F_{0}} \int_{0}^{2 \pi} \int_{\pi / 2}^{\pi} F\left(R_{2} \cos \theta\right) R_{2}^{2} \sin \theta d \theta d \phi \\
& =\pi R_{2}{ }^{2} \frac{2\left[\left(2 m_{0 i} k R_{2}-1\right) \exp \left(2 m_{0 i} k R_{2}\right)+1\right]}{\left(2 m_{0 i} k R_{2}\right)^{2}} .
\end{aligned}
$$

Note that the expression of the total incident radiation flux intercepted by a sphere in an absorbing medium has been given by Mundy et al. ${ }^{9}$ Evidently, the interception cross section depends not only on the particle geometric projected-area but also on the dielectric properties of the host medium as well as on the incident wavenumber.

The total energy scattered by the particle, prior to its attenuation due to the absorption by the host medium, can be obtained by integrating the radial component of the Poynting vector associated with the scattered wave on the particle surface. Thus, the corresponding inherent scattering cross section defined with respect to the reference irradiance $F_{0}$ can be given as follows:

$$
\begin{gathered}
\sigma_{s}=\frac{1}{F_{0}} \int_{0}^{2 \pi} \int_{0}^{\pi} \frac{c}{8 \pi} \operatorname{Re}\left\{\left[\vec{E}_{s}\left(\phi, \theta, R_{2}\right) \times \bar{H}_{s}^{*}\left(\phi, \theta, R_{2}\right)\right] \cdot \hat{r}\right\} R_{2}^{2} \sin \theta d \theta d \phi \\
=\frac{1}{F_{0}} \int_{0}^{2 \pi} \int_{0}^{\pi} \frac{c}{8 \pi} \operatorname{Re}\left[E_{s \theta}(\phi, \theta, R) H_{s \phi}^{*}\left(\phi, \theta, R_{2}\right)-E_{s \phi}\left(\phi, \theta, R_{2}\right) H_{s \theta}^{*}\left(\phi, \theta, R_{2}\right)\right] R_{2}^{2} \sin \theta d \theta d \phi \\
\left.=\frac{2 \pi}{m_{0 r} k^{2}} \operatorname{Im}\left\{\frac{1}{m_{0}} \sum_{n=1}^{\infty}(2 n+1)\left[\left|a_{n}\right|^{2} \xi_{n}^{\prime}\left(m_{0} k R_{2}\right) \xi_{n}^{*}\left(m_{0} k R_{2}\right)-\left|b_{n}\right|^{2} \xi_{n}\left(m_{0} k R_{2}\right) \xi_{n}^{\prime *}\left(m_{0} k R_{2}\right)\right]\right\},(8)\right\}
\end{gathered}
$$


where $\hat{r}$ is a unit vector along radial direction. The symbols, $\operatorname{Re}\{\}$ and $\operatorname{Im}\{\}$, indicate the real and imaginary parts of an argument, respectively. Similarly, the absorption cross section defined with respect to $F_{0}$ can be obtained as follows:

$$
\begin{gathered}
\sigma_{a}=-\frac{1}{F_{0}} \int_{0}^{2 \pi} \int_{0}^{\pi} \frac{c}{8 \pi} \operatorname{Re}\left\{\left[\vec{E}_{i}\left(\phi, \theta, R_{2}\right)+\vec{E}_{s}\left(\phi, \theta, R_{2}\right)\right] \times\left[\vec{H}_{i}^{*}\left(\phi, \theta, R_{2}\right)\right.\right. \\
\left.\left.+\vec{H}_{s}^{*}\left(\phi, \theta, R_{2}\right)\right] \cdot \hat{r}\right\} R_{2}^{2} \sin \theta d \theta d \phi \\
=-\frac{1}{F_{0}} \int_{0}^{2 \pi} \int_{0}^{\pi} \frac{c}{8 \pi} \operatorname{Re}\left\{\left[E_{i \theta}\left(\phi, \theta, R_{2}\right)+E_{s \theta}\left(\phi, \theta, R_{2}\right)\right] \cdot\left[H_{i \phi}^{*}\left(\phi, \theta, R_{2}\right)+H_{s \phi}^{*}\left(\phi, \theta, R_{2}\right)\right]\right. \\
\left.-\left[E_{i \phi}(\phi, \theta, R)+E_{s \phi}\left(\phi, \theta, R_{2}\right)\right] \cdot\left[H_{i \theta}^{*}\left(\phi, \theta, R_{2}\right)+H_{s \theta}^{*}\left(\phi, \theta, R_{2}\right)\right]\right\} R_{2}^{2} \sin \theta d \theta d \phi \\
=\frac{2 \pi}{m_{0 r} k^{2}} \operatorname{Im}\left\{\frac { 1 } { m _ { 0 } } \sum _ { n = 1 } ^ { \infty } ( 2 n + 1 ) \left[\psi_{n}\left(m_{0} k R_{2}\right) \psi_{n}^{\prime *}\left(m_{0} k R_{2}\right)-\psi_{n}^{\prime}\left(m_{0} k R_{2}\right) \psi_{n}^{*}\left(m_{0} k R_{2}\right)\right.\right. \\
+a_{n} \xi_{n}^{\prime}\left(m_{0} k R_{2}\right) \psi_{n}^{*}\left(m_{0} k R_{2}\right)-b_{n} \xi_{n}^{\prime}\left(m_{0} k R_{2}\right) \psi_{n}^{\prime}\left(m_{0} k R_{2}\right) \\
+a_{n}^{*} \psi_{n}^{\prime}\left(m_{0} k R_{2}\right) \xi_{n}^{*}\left(m_{1} k R\right)-b_{n}^{*} \psi_{n}\left(m_{0} k R_{2}\right) \xi_{n}^{*}\left(m_{0} k R_{2}\right) \\
\left.-\left|a_{n}\right|^{2} \xi_{n}^{\prime}\left(m_{0} k R_{2}\right) \xi_{n}^{*}\left(m_{0} k R_{2}\right)+\left|b_{n}\right|^{2} \xi_{n}\left(m_{0} k R_{2}\right) \xi_{n}^{\prime *}\left(m_{0} k R_{2}\right)\right\} .
\end{gathered}
$$

The extinction cross section $\sigma_{e}$ corresponding to the scattering and absorption cross sections in Eqs. (8) and (9), as is the same as its conventional definition when the host medium is nonabsorptive, is the summation of absorption and scattering cross section, that is

$$
\sigma_{e}=\sigma_{a}+\sigma_{s}
$$

Note that mathematical expressions that are similar to Eqs. (8) and (9) but in terms of absorbed and scattered energy have been presented by Sudiarta and Chylek ${ }^{11}$ in the case for a homogeneous sphere. To derive the energy absorbed by a homogeneous sphere in 
an absorbing medium, Fu and Sun ${ }^{12}$ used the internal field on the particle surface that is approached from the inside of the particle. It should be pointed out that the approach based on the internal field is more complicated than that based on the fields outside the particle in the case for a coated sphere. The increased complexity occurs because the internal field for a coated sphere, as is shown in Bohren and Huffman ${ }^{5}$, is in the form of

$$
\vec{E}_{t}=\sum_{n=1}^{\infty} E_{n}\left[f_{n} \vec{M}_{o l n}^{(1)}-i g_{n} \vec{N}_{e l n}^{(1)}+v_{n} \vec{M}_{o 1 n}^{(2)}-i w_{n} \vec{N}_{e l n}^{(2)}\right]
$$

whereas the counterpart of the preceding expression in the case for a homogeneous sphere is in the form of

$$
\vec{E}_{t}=\sum_{n=1}^{\infty} E_{n}\left[c_{n} \vec{M}_{o l n}^{(1)}-i d_{n} \vec{N}_{e l n}^{(1)}\right]
$$

In Eqs. (10) and (11), $\bar{M}_{o, e l n}^{(1,2)}$ and $\bar{N}_{o, e l n}^{(1,2)}$ are vector spherical harmonics whose detailed definitions can be found in van de Hulst ${ }^{2}$ and also in Bohren and Huffman ${ }^{5}$. We note that that the numerical computation of the four coefficients in Eq. (10) is much more complicated than the computation of the two coefficients in Eq. (11).

We apply the conventional definitions of the scattering and extinction efficiencies to the cross sections given by Eqs. (9) and (10) and define formality efficiency factors as follows:

$$
\begin{aligned}
& Q_{e}=\sigma_{e} /\left(\pi R_{2}^{2}\right), \\
& Q_{s}=\sigma_{s} /\left(\pi R_{2}^{2}\right) .
\end{aligned}
$$

Furthermore, we introduce the interception efficiency that is defined as

$$
Q_{i}=\sigma_{i} /\left(\pi R_{2}^{2}\right)
$$


where $\sigma_{i}$ is the interception cross section given by Eq. (7). It can be proven that the interception efficiency approaches to unity if the absorption of the host medium reduces to zero. In this circumstance, the scattering and extinction efficiencies defined in Eq. (12a) and (12b) regain their physical meanings as in the conventional sense. Note that the preceding efficiencies can be unbounded for a large size parameter if the host medium is strongly absorptive because the cross sections are specified with respect to the incident irradiance at the particle center, as is evident from Eqs. (7)-(10).

When the host medium is absorptive, the true or inherent scattering efficienciy (hereafter, referred to as $Q_{s, \text { inh }}$ ) for the scattering particle should be defined as the ratio of the scattered energy to the incident energy intercepted by the particle. Similarly, the inherent extinction efficiency (hereafter, referred to as $Q_{e \text {,inh }}$ ) is the ratio of the total attenuated (scattered + absorbed) energy to the portion of the incident energy intercepted by the particle. Mathematically, these two inherent optical efficiencies and their ratio (i.e., the inherent single-scattering albedo) can be expressed as follows:

$$
\begin{aligned}
& Q_{e, \text { inh }}=Q_{e} / Q_{i}, \\
& Q_{s, \text { inh }}=Q_{s} / Q_{i}, \\
& \omega_{0, \text { inh }}=Q_{s, \text { inh }} / Q_{e, \text { inh }}=Q_{s} / Q_{e},
\end{aligned}
$$

where $Q_{e}, Q_{s}$, and $Q_{i}$ are defined in Eqs. (12a), (12b) and (13), respectively. It is worth noting that the extinction and scattering efficiencies reported in Sudiarta and Chylek ${ }^{11}$ and also in Fu and $\operatorname{Sun}^{12}$ are the inherent quantities, $Q_{e \text {,inht }}$ and $Q_{s, \text { inht }}$, defined in Eqs. (14a) and (14b). Evidently, the inherent efficiencies do not have the conventional meanings in that the corresponding cross sections are not the products of the efficiencies and the geometric projected-area of the sphere. As a matter of fact, neither extinction nor 
scattering cross section may be defined without properly referencing a location for the incident irradiance when the host medium is absorptive.

The preceding inherent scattering efficiency is derived from the near field at the particle surface and is less useful in practice, because the scattered wave in the radiation zone is usually the relevant quantity. Thus, it is necessary to derive the apparent optical properties based on the far-field information. Using Eqs. (2e) and (2f) and the asymptotic form of the Riccati-Bessel functions, we can obtain the scattered field in radiation zone (or far field) that is given as follows:

$$
\begin{aligned}
& \left.E_{s \theta}(\phi, \theta, r)\right|_{m_{0}, k r \mid \rightarrow \infty}=\cos \phi \frac{\exp \left(i m_{0} k r\right)}{-i m_{0} k r} E_{0} S_{2}, \\
& \left.E_{s \phi}(\phi, \theta, r)\right|_{\left|m_{0} k r\right| \rightarrow \infty}=-\sin \phi \frac{\exp \left(i m_{0} k r\right)}{-i m_{0} k r} E_{0} S_{1},
\end{aligned}
$$

where the amplitude scattering functions $S_{1}$ and $S_{2}$ are given, respectively, by

$$
\begin{aligned}
& S_{1}=\sum_{n=1}^{\infty} \frac{2 n+1}{n(n+1)}\left[a_{n} \pi_{n}(\cos \theta)+b_{n} \tau_{n}(\cos \theta)\right], \\
& S_{2}=\sum_{n=1}^{\infty} \frac{2 n+1}{n(n+1)}\left[a_{n} \tau_{n}(\cos \theta)+b_{n} \pi_{n}(\cos \theta)\right] .
\end{aligned}
$$

Eqs. (15a) and (15b) are similar to their counterparts for the case when the host medium is nonabsorptive, except that the wavenumber in these expressions is scaled by the complex refractive index $m_{0}$. From Eqs. (15a) and (15b), the two components of scattered wave that are parallel and perpendicular, respectively, to the scattering plane can be expressed in a matrix form as follows:

$$
\left(\begin{array}{c}
E_{s / /}(\phi, \theta, r) \\
E_{s \perp}(\phi, \theta, r)
\end{array}\right)=\frac{\exp \left(i m_{0} k r\right)}{-i m_{0} k r}\left(\begin{array}{cc}
S_{2} & 0 \\
0 & S_{1}
\end{array}\right)\left(\begin{array}{c}
E_{i 0 / /} \\
E_{i 0 \perp}
\end{array}\right)
$$


where $\left(E_{s / /}, E_{s \perp}\right)=\left(E_{s \theta},-E_{s \phi}\right)$ and $\left(E_{i 0 / /}, E_{i 0 \perp}\right)=\left(E_{0} \cos \phi, E_{0} \sin \phi\right)$. As the scattered wave in the far-field region is a transverse wave, the magnetic field can be related to the corresponding electric field by an expression similar to Eq. (Ib). Thus, the irradiance associated with the scattered wave in the radiation zone or the far-field region is given by

$$
\begin{aligned}
& F_{s}(\phi, \theta, r)=\frac{c m_{0, r}}{8 \pi}\left(\left|E_{s, H}\right|^{2}+\left|E_{s, \perp}\right|^{2}\right) \\
& =\frac{c m_{0, r}}{8 \pi}\left|E_{0}\right|^{2} \frac{\exp \left(-2 m_{0, i} k r\right)}{\left|m_{0}\right|^{2} k^{2} r^{2}}\left(\left|S_{1}\right|^{2}+\left|S_{2}\right|^{2}\right) \\
& =\exp \left[-2 m_{0, i} k\left(r-R_{2}\right)\right] F_{0} \frac{\exp \left(-2 m_{0, i} k R_{2}\right)}{\left|m_{0}\right|^{2} k^{2} r^{2}}\left(\left|S_{1}\right|^{2}+\left|S_{2}\right|^{2}\right)
\end{aligned}
$$

In the preceding equation, the factor $\exp \left[-2 m_{1 i} k\left(r-R_{2}\right)\right]$ accounts for the absorption of the host medium in the region between particle and the observational point. The apparent scattering cross section of the particle, with respect to reference irradiance $F_{0}$, after it is traced back from the far-field scattered wave observed at a distance $r$ from the origin of the coordinate system, with an associated correction of the wave attenuation due to the absorption effect of the host medium, is given by

$$
\begin{aligned}
\tilde{\sigma}_{s} & =\frac{\exp \left[2 m_{0 i} k\left(r-R_{2}\right)\right]}{F_{0}} \int_{0}^{2 \pi} \int_{0}^{\pi} F_{s}(\phi, \theta, r) r^{2} \sin \theta d \theta d \phi \\
& =\frac{\exp \left(-2 m_{0 i} k R_{2}\right)}{\left|m_{0}\right|^{2} k^{2}} \int_{0}^{2 \pi} \int_{0}^{\pi}\left(\left|S_{1}\right|^{2}+\left|S_{2}\right|^{2}\right) \sin \theta d \theta d \phi \\
& =\frac{2 \pi \exp \left(-2 m_{0 i} k R_{2}\right)}{\left|m_{0}\right|^{2} k^{2}} \sum_{n=1}^{\infty}(2 n+1)\left(\left|a_{n}\right|^{2}+\left|b_{n}\right|^{2}\right) .
\end{aligned}
$$

A comparison of the scattering cross section in Eq. (18) to its counterpart in the case of a nonabsorptive host medium shows that they are similar except for an exponential factor $\exp \left(-2 m_{0 i} k R_{2}\right)$ along with a complex host medium refractive index $m_{0}$ involved in 
Eq.(18). Furthermore, the apparent scattering efficiency is quite different from its inherent counterpart, as is evident from the comparison between Eqs. (8) and (18). This discrepancy occurs because the host absorption over the distance between particle and an observational point in the radiation zone cannot be accounted for exactly by the exponential factor $\exp \left[2 m_{0 i} k\left(r-R_{2}\right)\right]$. The local plane-wave feature of the scattered wave, described by the exponential form for the spatial phase variation in Eq. (16), is valid only for the far-field regime. Thus, an assumption of exponential attenuation for the near-field region will overestimate or underestimate the absorption in the host medium. But this will not affect multiple scattering calculation as long as the apparent scattering cross section is used, though the host absorption is assumed to have an exponential form regardless of the location in the medium with respect to scattering particles.

In practice, it is critical to transform the incident Stokes vector to its scattered counterpart using the apparent single-scattering properties. Based on Eqs. (16) and (18) and the definition of Stokes vector ${ }^{2}$, the Stokes vector associated with scattered field is related to its incident counterpart via scattering phase matrix as follows:

$$
\begin{aligned}
\left(\begin{array}{l}
I_{s}(\phi, \theta, r) \\
Q_{s}(\phi, \theta, r) \\
U_{s}(\phi, \theta, r) \\
V_{s}(\phi, \theta, r)
\end{array}\right) & =\frac{\exp \left[-2 m_{0 j} k\left(r-R_{2}\right)\right] \tilde{\sigma}_{s}}{r^{2}} \\
& \cdot \frac{P_{11}}{4 \pi}\left[\begin{array}{cccc}
1 & P_{12} / P_{11} & 0 & 0 \\
P_{12} / P_{11} & 1 & 0 & 0 \\
0 & 0 & P_{33} / P_{11} & -P_{33} / P_{11} \\
0 & 0 & P_{43} / P_{11} & P_{33} / P_{11}
\end{array}\right]\left(\begin{array}{c}
I_{i 0} \\
Q_{i 0} \\
U_{i 0} \\
V_{i 0}
\end{array}\right),
\end{aligned}
$$


where $\left(I_{i 0}, Q_{i 0}, U_{i 0}, V_{i 0}\right)$ is the incident Stokes vector at the origin of the coordinate system. Note that the incident Stokes vector associated with a wave propagating along $z$ coordinate axis can be expressed for an arbitrary location $(\phi, \theta, r)$ in the form of

$$
\left(\begin{array}{l}
I_{i}(\phi, \theta, r) \\
Q_{i}(\phi, \theta, r) \\
U_{i}(\phi, \theta, r) \\
V_{i}(\phi, \theta, r)
\end{array}\right)=\exp \left(-2 m_{0 i} k r \cos \theta\right)\left(\begin{array}{c}
I_{i 0} \\
Q_{i 0} \\
U_{i 0} \\
V_{i 0}
\end{array}\right) .
$$

In Eq. (19) $P_{11}$ is the normalized phase function in the sense that $\left[\int_{0}^{\pi} P_{11}(\cos \theta) \sin \theta d \theta\right] / 2$ is unity. From the far-field perspective, the parameter $\tilde{\sigma}_{s}$ is the scattering cross section. For this reason, we refer to $\tilde{\sigma}_{s}$ as the apparent scattering cross section as viewed from a point away from the particle. The apparent scattering cross section should be used in any radiative transfer calculations dealing with the far-field. The nonzero elements of the phase matrix in Eq. (20) take the same form as for the case for non-absorbing host medium and are given by

$$
\begin{aligned}
& P_{11}=\frac{\left|S_{1}\right|^{2}+\left|S_{2}\right|^{2}}{\sum_{n=1}^{\infty}(2 n+1)\left(\left|a_{n}\right|^{2}+\left|b_{n}\right|^{2}\right)}, \\
& P_{12} / P_{11}=\frac{\left|S_{2}\right|^{2}-\left|S_{1}\right|^{2}}{\left|S_{2}\right|^{2}+\left|S_{1}\right|^{2}}, \\
& P_{33} / P_{11}=\frac{2 \operatorname{Re}\left(S_{1} S_{2}^{*}\right)}{\left|S_{2}\right|^{2}+\left|S_{1}\right|^{2}}, \\
& P_{43} / P_{11}=\frac{2 \operatorname{Im}\left(S_{1} S_{2}^{*}\right)}{\left|S_{2}\right|^{2}+\left|S_{1}\right|^{2}} .
\end{aligned}
$$

The asymmetry factor has the same form for either the absorptive and non-absorptive host medium, as is evident from the comparison of the results presented by Kerker ${ }^{3}$ and 
Fu and Sun ${ }^{12}$. In addition, the asymmetry factor for a coated sphere can be expressed in the same form as that for a homogeneous sphere, given by

$$
\begin{gathered}
g=\frac{1}{2} \int_{0}^{\pi} P_{11}(\cos \theta) \cos \theta \sin \theta d \theta \\
=\frac{2 \sum_{n=2}^{\infty}\left\{\operatorname{Re}\left[(n-1)(n+1)\left(a_{n-1} a_{n}^{*}+b_{n-1} b_{n}^{*}\right) / n\right]+(2 n-1) /[(n-1) n] \operatorname{Re}\left(a_{n-1} b_{n-1}^{*}\right)\right\}}{\sum_{n=1}^{\infty}(2 n+1)\left(\left|a_{n}\right|^{2}+\left|b_{n}\right|^{2}\right)} .
\end{gathered}
$$

For the absorption within the particle, we do not distinguish between the apparent and inherent features. Thus, the apparent extinction cross section associated with the $\tilde{\sigma}_{s}$ can be defined as follows:

$$
\tilde{\sigma}_{e}=\sigma_{a}+\tilde{\sigma}_{s}
$$

where the absorption cross section is that defined in Eq. (9). As is similar to the case for inherent optical properties, the apparent scattering and extinction efficiencies can be defined as follows:

$$
\begin{aligned}
& \tilde{Q}_{e}=\tilde{\sigma}_{e} /\left(\pi R_{2}^{2}\right), \\
& \tilde{Q}_{s}=\tilde{\sigma}_{s} /\left(\pi R_{2}^{2}\right) .
\end{aligned}
$$

The apparent extinction and scattering efficiencies as well as single-scattering albedo that are defined with respect to the truly intercepted incident irradiance are given by

$$
\begin{aligned}
& Q_{e, \mathrm{app}}=\tilde{Q}_{e} / Q_{i}, \\
& Q_{s, \mathrm{app}}=\tilde{Q}_{s} / Q_{i}, \\
& \omega_{0, \mathrm{app}}=Q_{s, \mathrm{app}} / Q_{e, \mathrm{app}}=\tilde{Q}_{s} / \tilde{Q}_{e} .
\end{aligned}
$$


The preceding apparent scattering efficiency is essentially the unattenuated scattering efficiency defined by Mundy et al. ${ }^{9}$ in the case of homogeneous sphere. However, the extinction efficiency defined by those authors can be smaller than the scattering efficiency. This shortcoming is overcome by the present definition of apparent extinction efficiency.

C. Proper Form of Single-Scattering Properties for Multiple Scattering by a Polydisperse Particle System within Absorbing Medium

In this section we present the radiative transfer equation derived for a polydispersive system in an absorbing medium based on the apparent single-scattering properties. For simplicity without losing generality, we consider the scalar radiative transfer equation. That is, we do not account for the full Stokes vector but only the intensity of radiation. Thus, according to Eq. (19), for the scattering process associated with an individual particle with a radius $R$, we can express the scattered intensity as

$$
I_{s}(\phi, \theta, r)=\frac{\exp \left[-2 m_{0 i} k(r-R) \tilde{\sigma}_{s}\right.}{r^{2}} \frac{P_{11}}{4 \pi} I_{i 0},
$$

where $\tilde{\sigma}_{s}$ is the apparent scattering cross section defined in Eq. (18). In Eq. (26), the transformation of incident intensity to the scattered intensity explicitly involves the particle size, $R$. This is a shortcoming that prevents the applicability of Eq. (26) to multiple scattering processes involving a polydisperse particle system. To circumvent this disadvantage, we define the scaled apparent cross section as follows

$$
\tilde{\sigma}_{s, \text { scaled }}=\exp \left(2 m_{0} k R\right) \tilde{\sigma}_{s} ;
$$

and consequently, the scaled extinction cross section by 


$$
\tilde{\sigma}_{e, \text { scaled }}=\sigma_{a}+\tilde{\sigma}_{s, \text { scaled }}
$$

With these definitions, Eq. (26) can be simplified as follows:

$$
I_{s}(\phi, \theta, r)=\frac{\exp \left(-2 m_{0 i} k r\right) \tilde{\sigma}_{s, \text { scaled }}}{r^{2}} \frac{P_{11}}{4 \pi} I_{i 0} .
$$

In Eq. (28) the incident intensity is specified at the center of particle. Through the use of the scaled scattering cross section, the absorption path for two subsequential scattering event processes is measured between the centers of particles. This feature substantially simplifies the radiative transfer calculation in absorbing medium.

For a ploydisperse particle system, let us assume the volume-normalized concentration for the particle at a location within the host medium to locally be $N(\bar{s}, R)$, where $R$ is the radius of the particle and $\bar{s}$ is the position vector. The extinction and scattering coefficients due to the effect of particle scattering and absorption as viewed from the far-field perspective, rather than the absorption of the host medium, can be given by the particle's scaled apparent scattering properties as follows:

$$
\begin{aligned}
& \tilde{\beta}_{e, \mathrm{p}}(\vec{s})=\int_{R_{\min }}^{R_{\max }} \tilde{\sigma}_{e, \text { scaled }}(\vec{s}, R) N(\vec{s}, R) d R, \\
& \tilde{\beta}_{s, \mathrm{p}}(\vec{s})=\int_{R_{\min }}^{R_{\max }} \tilde{\sigma}_{s, \text { scaled }}(\vec{s}, R) N(\vec{s}, R) d R,
\end{aligned}
$$

where the subscript $p$ indicates that the extinction and scattering coefficients are for particles. The quantity $N(\vec{s}, R)$ has units of number/volume/length and $\tilde{\sigma}_{e, \text { scaled }}$ (also $\left.\tilde{\sigma}_{s, \text { scaled }}\right)$ has units of area. Thus, both $\tilde{\beta}_{e, \mathrm{p}}(\vec{s})$ and $\tilde{\beta}_{s, \mathrm{p}}(\vec{s})$ have units of inverse length.

In the present formulation we ignore the Rayleigh scattering by the molecules of the host medium for the sake of simplicity, which, in principle, can be treated in the same 
manner as that for particulate inclusions in the medium. The extinction coefficient for the host medium is due solely to the absorption of the medium and is given by

$$
\beta_{e, \text { host }}(\vec{s})=2 m_{0 i}(\vec{s}) k \text {. }
$$

Based on the fundamental principle of radiative transfer (e.g., Chandrasekar ${ }^{15}$ ) and Eqs. (18a), (18b), and (19), the radiative transfer equation for the multiple scattering process occurring in an absorbing medium can then be written as follows:

$$
(\hat{\Omega} \cdot \nabla) I(\hat{\Omega}, \vec{s})=-\left[\beta_{e, p}(\vec{s})+\beta_{e, \text { host }}(\vec{s})\right] I(\hat{\Omega}, \vec{s})+J(\hat{\Omega}, \vec{s}),
$$

where $\hat{\Omega}$ is a unit vector specifying the propagating direction of the radiation. The first term on the right-hand side of the equation corresponds to the attenuation of radiation by particle extinction and host medium absorption. $J(\hat{\Omega}, \vec{s})$ is the source function arising from the multiple scattering by the particles, given by

$$
J(\hat{\Omega}, \vec{s})=\frac{\tilde{\beta}_{s, \mathrm{p}}(\vec{s})}{4 \pi} \int_{4 \pi} I(\hat{\Omega}, \vec{s}) P_{11}\left(\vec{s}, \hat{\Omega}, \hat{\Omega}^{\prime}\right) d \hat{\Omega}^{\prime}
$$

In Eqs. (31a) and (31b), we do not distinguish between the direct and diffuse radiation. In this form of the radiation transfer equation, the source of the radiation can be implemented through a boundary condition (e.g., Preisendorfer and Mobely ${ }^{16}$ ). In practice, numerical accuracy is achieved by separating the diffuse and direct components of radiation. Situations exist where it is difficult to distinguish between the diffuse and direct compoents of the radiation, such as for the solution of the radiation field inside a water layer with a wavy surface. In Eq. $(31 \mathrm{~b})$, the phase function for the polydisperse system is given by the mean value obtained from the average particle phase function integrated over a specified particle size distribution and takes the form of 


$$
P_{11}\left(\vec{s}, \hat{\Omega}, \hat{\Omega}^{\prime}\right)=\frac{\int_{R_{\min }}^{R_{\max }} \tilde{\sigma}_{s, \text { scaled }}(\vec{s}, R) P_{11}(\vec{s}, R, \hat{\Omega}, \hat{\Omega}) N(\vec{s}, R) d R}{\int_{R_{\min }}^{R_{\max }} \tilde{\sigma}_{s, \text { caled }}(\vec{s}, R) N(\vec{s}, R) d R} .
$$

It is straightforward to use the scaled apparent single-scattering properties in various radiative transfer computational methods even for those that are not explicitly based on the radiative transfer equation. For example, in the Monte Carlo method involving an absorbing host medium, one can use $\tilde{\beta}_{e, \mathrm{p}}(\bar{s})$ to determine the mean freepath that a photon encounters next scattering or absorption event in a manner as in the conventional method. For a scattering or absorption event, one can use the ratio of $\tilde{\beta}_{s, \mathrm{p}}(\bar{s})$ to $\tilde{\beta}_{e, \mathrm{p}}(\bar{s})$ to determine whether the photon is scattered or absorbed. The new propagating direction of the scattered photon may be determined from the phase function according to a conventional algorithm. The effect of the absorption of the host medium is that the weight for the photon is scaled by a factor of $\exp \left(-2 m_{0 i} k d\right)$ between two successive scattering (or absorption) events, where $d$ is the distance between the two events or, alternatively, the free-path length. Furthermore, in dealing with multiple scattering in an absorptive host medium, weighting procedures for the bulk singlescattering properties may be a candidate for computational convenience (e.g., Tsay et al. ${ }^{17}$, by Platnick and Valero ${ }^{18}$ )

\section{Numerical Results and Discussions}

Based on the analyses in the previous section, we have developed a computational program to solve for the inherent and apparent optical efficiencies and the full phase matrix for a coated sphere within an absorbing medium. The previous codes written by 
Wiscombe ${ }^{4}$, Bohren and Huffman ${ }^{5}$, and Toon and Ackerman ${ }^{8}$ for spheres in nonabsorbing medium were of great benefit to the development of the present code. In particular, we find that the convergence criterion suggested by Wiscombe ${ }^{4.7}$ is useful for computational efficiency and accuracy. The present code has been validated via comparison with the previous inherent optical efficiencies and phase function of homogeneous spheres in an absorbing medium reported by Sudiarta and Chylek ${ }^{11}$ and Fu and Sun ${ }^{12}$. Additionally, the results obtained from the present code agree well with those from the conventional Lorenz-Mie calculations when the absorption of host medium is reduced to zero.

Figure 2 shows the inherent and apparent extinction efficiency, single-scattering albedo, and asymmetry factor for spheres of soot embedded within ice at $1.38,3.75$, and $11 \mu \mathrm{m}$. The complex refractive indices of ice and soot at the three wavelengths are obtained by interpolation of Warren's data ${ }^{19}$ for ice and d'Almeida et al.'s data ${ }^{20}$ for soot and are listed in Table 1. These three wavelengths were chosen because they are commonly used in airborne or satellite retrieval. The $1.38 \mu \mathrm{m}$ band is very effective for detecting cirrus clouds. The single-scattering properties of soot or air bubbles within ice are interesting to the study of cirrus clouds. Ice crystals within cirrus clouds may contain black carbon coming from biomass burning and has not been fully explored. Furthermore, ice crystals may contain pockets of air bubbles; this effect has also been ignored to date in the computation of single scattering properties. To solve for the optical properties of ice crystals with impurities on the basis of ray-tracing technique (e.g., Macke et al. ${ }^{21}$, Labonnote et al. ${ }^{22}$ ), Lorenz-Mie calculation is carried out for the inclusions that are assumed to be very small and the surrounding ice medium is treated as unbound. From Fig. 2, the inherent and apparent values are essentially the same at $1.38 \mu \mathrm{m}$ for 
either the extinction efficiency or the single-scattering albedo because the absorption of ice is negligible at this wavelength. However, substantial differences between the inherent and apparent optical properties are noted at 3.75 and $11 \mu \mathrm{m}$, in particular, for the case of the single-scattering albedo at $11 \mu \mathrm{m}$. From Fig. 2, one may notice that the apparent scattering cross section is smaller than its inherent counterpart. Figure 2 also presents the asymmetry factor for the phase function - note that the asymmetry factor can be negative. In addition, the inherent extinction efficiency converges to 1 instead of 2 . These two features associated with the optical properties of a sphere in an absorbing medium have been reported by Sudiarta and Chylek ${ }^{11}$, and Fu and Sun ${ }^{12}$.

Figure 3 is similar to Fig. 2, except that the scatter in the former case is a void (air bubble) in the medium. Because the air bubble is nonabsorbing, both inherent and apparent single-scattering albedo is unity. For the extinction efficiency, significant differences are evident from a comparison of the apparent and inherent optical properties at 3.7 and $11.0 \mu \mathrm{m}$, wavelengths at which ice is strongly absorptive. For the asymmetry factor shown in Fig. 3, negative values are not observed. When the host medium is strongly absorptive, the diffraction wave that contributes to forward scattering is essentially suppressed. If the scattering particle is also strongly absorptive, there is no transmittance through the particle to contribute to forward scattering. In this case the backscattering can be larger than forward scattering, leading to negative asymmetry factor. Contrary to this argument, for the air bubble case shown in Fig. 3, there still is a significant amount of radiation transmitted through the air bubble and the forward scattering is stronger than backscattering. 
To convert the inherent or apparent extinction or scattering efficiency to the corresponding cross section, the interception efficiency factor $Q_{i}$ is required, as is evident from Eqs. (14a)-(14b), and (25a)-(25b). Figure 4 shows the interception efficiency for three wavelengths with ice as the host medium. At $1.38 \mu \mathrm{m}$ wavelength, the absorption of ice is negligible, and $Q_{i}$ is essentially unity. However, for the two absorbing wavelengths the interception efficiency can be very large. In particular, at $11 \mu \mathrm{m}$, the $Q_{i}$ factor can be unbounded when the particle size increases. This occurs because the interception factor is defined with respect to the incident irradiance at the particle center. For a strongly absorptive host medium, the incident irradiance can be attenuated substantially within a distance on the order of the particle radius that is comparable to the incident wavelength.

Figure 5 shows the complete nonzero elements of phase matrix for homogeneous spheres within an absorbing media. For the host medium, the refractive index is selected as $1.0+\mathrm{i} 0.0,1.0+\mathrm{i} 0.01$, and $1.0+\mathrm{i} 0.05$, corresponding to the values used in Sudiarta and Chylek ${ }^{11}$. The particle refractive index is selected as $1.33+\mathrm{i} 0.0$ that is essentially the refractive index of water droplet at a visible wavelength. Obviously, the absorption of the host medium substantially influences the scattering phase matrix of the particles, particularly for the large size parameter. For the moderate size parameter $X=50$ and large size parameter $X=500$, the scattering of the sphere is subtantially reduced in sidescattering directions when the host absorption is strong, as is evident from the phase functions shown. For scattering by an individual sphere, the resonant effect is significant for the phase matrix elements associated with polarization. The resonant effect is particularly pronounced for the phase elements associated with the polarization configuration. Recently, using modern visualization techniques, Mishchenko and Lacis ${ }^{23}$ 
invistigated the morphology-dependent resonances for homogeneous spheres within a nonabsorbing host medium. In future work, it would be interesting to study the impact of the host absorption on the resonances in a manner suggested by those authors.

To smooth out the resonant fluctuations, we include a size distribution in the scattering calculation. Figure 6 shows the phase matrix for three mean size parameters: 10 with a binwidth of 5 to 15,50 with a binwidth of 40 to 60 , and 500 with a binwidth of 450-550. The effect of host absorption is pronounced. For very strong absorbing host medium, the phase function value is significantly reduced in forward and sidescattering directions. In particular, the rainbow feature associated within a scattering by spheres is smoothed out by the host absorption, as is seen from the phase functions shown for the case of $\langle X\rangle=500$. The effect of the host absorption on the polarization configuration of the scattered wave is also evident, which is particular significant for $P_{33} / P_{1 /}$ and $-P_{12} / P_{I l}$.

Figure 7 is similar to Fig. 6, except that the scattering particle is soot for the former. For the moderate and large size parameters, i.e., $<\mathrm{X}>=50$ and 500 , the phase function values are larger for backscattering directions than for either forward and sidescattering directions when the host absorption is large. This occurs because soot is a strongly absorbing medium and does not allows the transmission of the incident radiation whereas the host absorption suppresses the diffraction peak. From Fig. 7, it can also be noted that the host absorption has a very minor effect on polarization. Since soot is very absorptive, the external reflection occurring at the particle surface dominates the scattered field. The polarization configuration of the externally reflected wave is not sensitive to particle size and the host absorption. 
Black carbon in the atmosphere may serve as nuclei for cloud droplets and modifies the bulk radiative properties of clouds. Figure 8 shows the inherent and apparent extinction efficiency and single-scattering albedo for water droplets containing soot for weak and strong host absorption conditions. The ratio of the radius of soot core to the radius of water shell has been selected as $R_{1} / R_{2}=0.1,0.5$, and 0.9 . For extinction efficiency, the effect of the impurity on water droplets is primarily in the size parameter region of 1-20 since the extinction efficiency will approach its asymptotic value when size parameter is large. The effect of the impurity on single-scattering, however, is pronounced for the entire size parameter spectrum shown. Even a small amount of soot added to water droplets can substantially reduce the single-scattering albedo, as is evident from the case for $R_{1} / R_{2}=0.1$. For weak host absorption values, the single-scattering albedo tends to reach its asymptotic value when the size parameter is large. On the contrary, the single-scattering albedo decrease with increasing size parameter in the large size parameter regime if a strong host absorption is involved.

Figure 9 is similar to Fig. 8, except that Fig. 9 shows results for ice spheres containing air bubbles. Because the imaginary part of ice is small and air bubble is nonabsorptive, both inherent and apparent single-scattering albedo is essentially unity regardless of the thickness of ice shell of the particles. From the extinction efficiencies shown, the extinction maximum is shifted toward large size parameters when the thickness of the ice shell decreases. In addition, the host absorption reduces the asymptotic values of the extinction efficiency in a manner similar to the case for homogeneous sphere. 
Figures 10 and 11 show the nonzero elements of phase matrix for soot spheres coated with water shell and for ice spheres containing air bubbles, respectively. For both cases, the refractive index of the host medium is assumed as $1.0+10.01$. A significant sensitivity to the ratio of core size to the shell size is demonstrated for the singlescattering properties. For the coated black carbon, the polarization configuration for the case when black carbon is dominant (i.e., $R_{1} / R_{2}=0.9$ ) is quite different from the other two cases for which the water is the dominant component of the scatterer. Similarly, for the case of a hollow ice sphere, the polarization feature is unique when the ice shell is thin and particle size is large.

\section{Summary and Conclusions}

We have extended the conventional Lorenz-Mie formalism to the scattering process associated with a coated sphere embedded within an absorbing medium. We have clarified that there are two ways of deriving the scattering and extinction cross sections. The scattering cross section derived from the near-field on the particle surface is the inherent optical properties of the particles, which is less useful in practice. Alternatively, the scattering cross section may be derived from the far-field wave with a proper scaling of the host medium absorption over the distance between particle and the location where the far-field is specified. This defines the apparent optical properties that implicitly contain the host absorption information in near-field regime. We show that the mathematical expressions for the inherent and apparent scattering properties are quite different. Furthermore, we have developed a computational code to compute the inherent and apparent extinction efficiencies, single-scattering albedos, and asymmetry factors as well as the complete phase matrix. 
Numerical calculations for spheres in absorbing media show that apparent and inherent optical properties can be different by several tens of percent if the host absorption is strong. The difference, however, reduces to zero if the host absorption is negligible. If the scattering particle is transparent (i.e., the imaginary part of the refractive index is zero), the host absorption has a significant impact of the nonzero elements of the phase matrix associated with the polarization configuration of scattered wave. We find that the effect of host absorption on the polarization effect is small if the particle itself is also strongly absorptive even if the host medium is substantially absorptive. Two specific applications discussed were of the scattering characteristics of (a) black carbon coated with water and (b) hollow ice spheres, i.e., ice spheres containing air bubbles. We found that black carbon included in water droplets can substantially reduces the singlescattering albedo for small size parameter even if the amount of the impurity is small. For hollow ice spheres, the single-scattering albedo is essentially unity regardless of host absorption. However, the variation pattern of the extinction efficiency versus size parameter can be substantially changed by strong host absorption.

In the case of a large size parameter $(<\mathrm{X}>=500)$ for coated soot or hollow ice spheres, the polarization elements of the phase matrix are unique in that $-P_{12} / P_{11}$ approaches unity whereas $P_{33} / P_{11}$ reduces to zero for scattering angles between $10-80^{\circ}$ if the particle shells are thin. Finally, we have defined a proper form of single-scattering properties for application to multiple scattering computation. It is shown that the conventional technique for radiative transfer calculations can be applied if the scaled apparent single-scattering properties are used. 
This research is supported by the NASA Radiation Sciences Program managed by Dr. Don Anderson, and partially supported by MODIS, PICASSO-CENA, and Wisconsin GIFTS/IOMI-MURI project, the Office of Naval Research, and the Atmospheric Radiation Measurement (ARM) program sponsored by the U.S. Department of Energy (DOE) under Contract DE-AI02-00ER62901. 


\section{References}

1. G. Mie, "Beigrade zur optik truber medien, speziell kolloidaler metallosungen," Ann. Phys. (Leipzip) 25, 377-455 (1908).

2. H. C. van de Hulst, Light Scattering by Small Particles, (Wiley, New York, 1957).

3. M. Kerker, The Scattering of Light and Other Electromagnetic Radiation, (Academic, New York, 1963).

4. W. J. Wiscombe, "Mie scattering calculation," NCAR Technical Note TN-140+STR (National Center for Atmospheric Research, Boulder, Colo., 1979).

5. C. F. Bohren and D. R. Huffman, Absorption and Scattering of Light by Small Particles, (Wiley, New York, 1983).

6. J. Dave, "Subroutines for computing the parameters of the electromagnetic radiation scattered by a sphere," IBM Report No. 320-3237 (IBM Scientific Center, Palo Alto, CA., 1968)

7. W.J. Wiscombe, "Improved Mie scattering algorithms," Appl. Opt., 19, 1505-1509 (1980).

8. O. B. Toon and T. P. Ackerman, "Algorithms for the calculation of scattering by starified spheres," Appl. Opt., 20, 3657-3660 (1981).

9. W. C. Mundy, J.A. Roux, and A. M. Smith, "Mie scattering by spheres in an absorbing medium," J. Opt. Soc. Am., 64, 1593-1597 (1974).

10. P. Chylek, "Light scattering by small particles in an absorbing medium," J. Opt. Soc. Am., 67, 561-563 (1977).

11. I. W. Sudiarta and P. Chylek, "Mie scattering formalism in absorbing medium," in the Proceedings of the Fifth Conference on Light Scattering by Nonspherical 
Particles: Theory, Measurements, and Applications. August 28-Septmber 1, 2000, Halifax, Canada (available from the US Army Research Laboratory, Adelphi, MD. 20783)

12.Q. Fu and W. Sun, "Mie theory for light scattering by a spherical particle in an absorbing medium," Appl. Opt., 40, 1354-1361 (2001).

13. W. D. Ross, "Computation of Bessel Functions in Light Scattering Studies,", Appl. Opt., 11, 1919-1923 (1972).

14.G. W. Kattawar and D. A. Hood, "Electromagnetic scattering from a spherical polydispersion of coated spheres," Appl. Opt. 15, 1996-1999 (1976).

15. S. Chandrasekhar, Radiative Transfer (Oxford University Press, London, 1950).

16.R. W. Preisendorfer and C. D. Mobely, "Direct and inverse irradiance models in hydrologic optics," Limnol. Oceanogr., 29, 903-929, 1984.

17. S.-C. Tsay, K. Stamnes, and K. Jayaweera, "Radiative energy budget in the cloudy and hazy arctic,"," J. Atmos. Sci., 46, 1002-1018 (1989).

18. S. Platnick, F. P.J. Valero, "A validation of a satellite cloud retrieval during ASTEX," J. Atmos. Sci., 15, 2985-3001 (1995).

19. S. Warren, "Optical constants of ice from the ultraviolet to the microwave," Appl. Opt. 23, 1206-1225 (1984).

20.G. A. d'Almeida, P. Koepke, and E. P. Shettle, Atmospheric Aerosols: Global Climatology and Radiative Characteristics (A. Deepak Publishing, Hampton, Virginia, 1991).

21. A. Macke, M. I. Mishchenko, and B. Cairns, "The influence of inclusions on light scattering by large ice particles," J. Geophys. Res., 101, 23,311-23,316, 1996. 
22. L. C. Labonnote, G. Brogniez, J.-C. Buriez, M. Doutriaux-Boucher, J. F. Gayet, and A. Macke, "Polarized light scattering by inhomogeneous hexagonal monocrystals: Validation with ADEOS-POLDER measurements," J. Geophys. Res. (in press).

23. M. I. Mishchenko and A. A. Lacis, 2000: Manifestations of morphology-dependent resonances in Mie scattering matrices, Appl. Math. and Comput. 116, 167-179. 
Table 1. The complex refractive index for soot and ice at three wavelengths, which are based on the interpolation of the data presented by Warren and d'Almeida for ice and soot, respectively.

\begin{tabular}{|c|c|c|}
\hline Wavelength $(\mu \mathrm{m})$ & Ice & Soot \\
\hline 1.38 & $1.2943+\mathrm{i} 1.580 \times 10^{-5}$ & $1.7804+\mathrm{i} 4.552 \times 10^{-1}$ \\
\hline 3.75 & $1.3913+\mathrm{i} 6.796 \times 10^{-3}$ & $1.9000+\mathrm{i} 5.700 \times 10^{-1}$ \\
\hline 11.0 & $1.0925+\mathrm{i} 2.480 \times 10^{-1}$ & $2.23+\mathrm{i} 7.300 \times 10^{-1}$ \\
\hline
\end{tabular}




\section{Figure Caption}

Figure 1. Geometry for scattering by a coated sphere embedded in an absorbing medium.

Figure 2. Inherent and apparent extinction efficiency and single-scattering albedo values for soot spheres embedded in an ice medium at wavelengths of $1.38,3.75$, and $11.0 \mu \mathrm{m}$. Also shown are the asymmetry factor values.

Figure 3. Same as Figure2, except that the scatterers are air bubbles in ice medium.

Figure 4. The interception efficiency defined for particles embedded in ice medium at three wavelengths.

Figure 5. The non-zero phase matrix elements for homogeneous (uncoated) spheres that are embedded in an absorbing media. Results are provided for three values of the imaginary refractive index and for three size parameters. The particle refractive index is assumed to be $1.33+\mathrm{i} 0.0$, which is essentially the refractive index for water at a visible wavelength.

Figure 6. Same as Figure 5 except for a multidisperse particle system.

Figure 7. Same as Figure 6, except that the particle refractive index is $1.75+\mathrm{i} 0.435$, which is the refractive index for soot at a visible wavelength.

Figure 8. Comparison of inherent and apparent extinction efficiency and singlescattering albedo for a soot sphere coated with water. The refractive indices for the host medium are chosen to be $1.0+\mathrm{i} 0.001$ and $1.0+\mathrm{i} 0.01$.

Figure 9. Same as Figure 8, except that the scattering particles are hollow ice spheres. 
Figure 10. Phase matrix elements for a particle of water-coated soot. The host refractive index is chosen as $1.0+10.01$.

Figure 11. Same as Figure 10, except for a hollow ice sphere. 


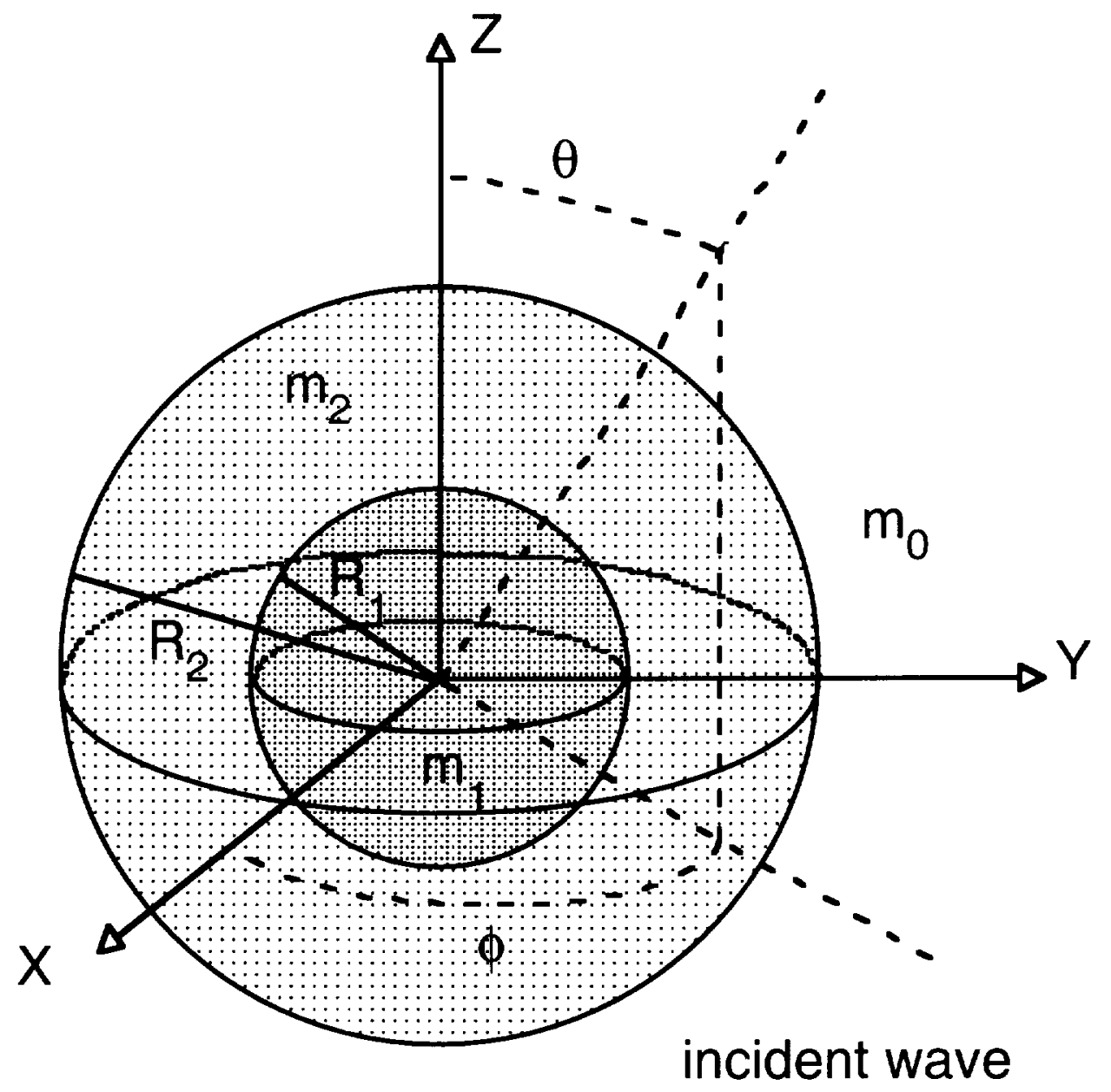

$\left.\hat{\uparrow} \hat{\uparrow} \hat{\mid}\right|_{E_{B}} \hat{H}_{H_{s}}$

Fig.1 
Inherent and Apparent Optical Properties of Soot Spheres in Ice
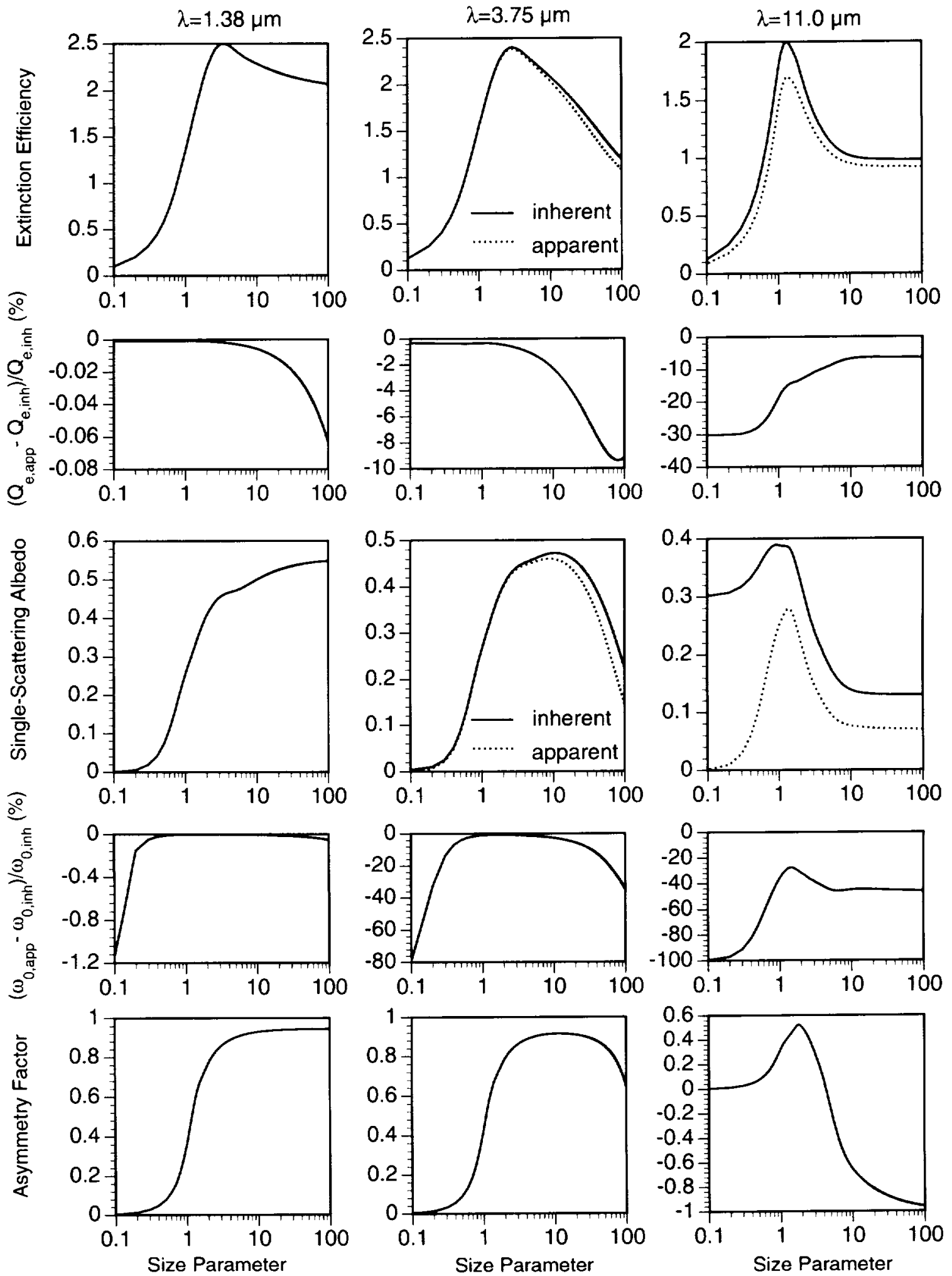

Fig.2 


\section{Inherent and Apparent Optical Properties of Air Bubbles in Ice}
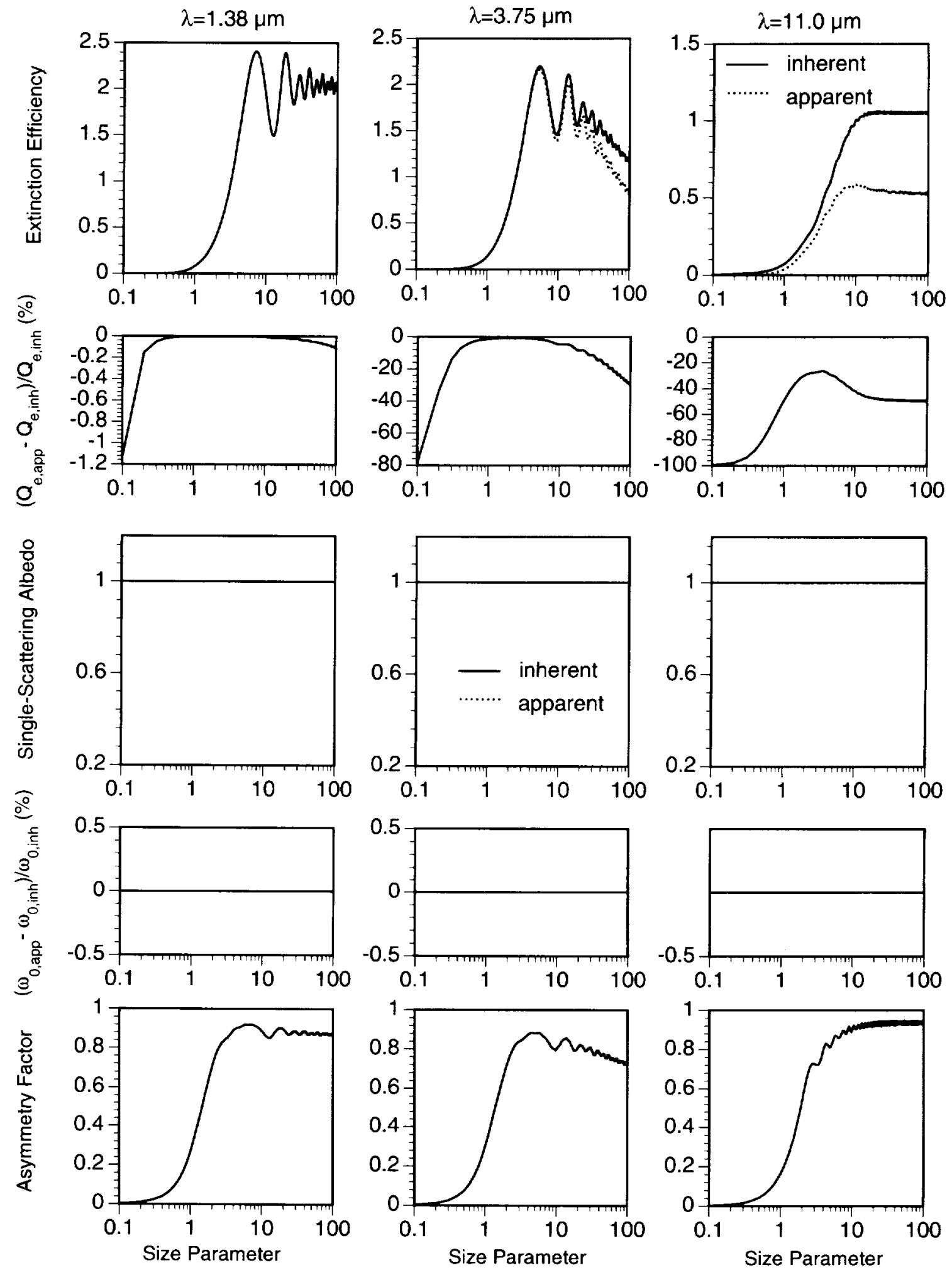

Fig.3 

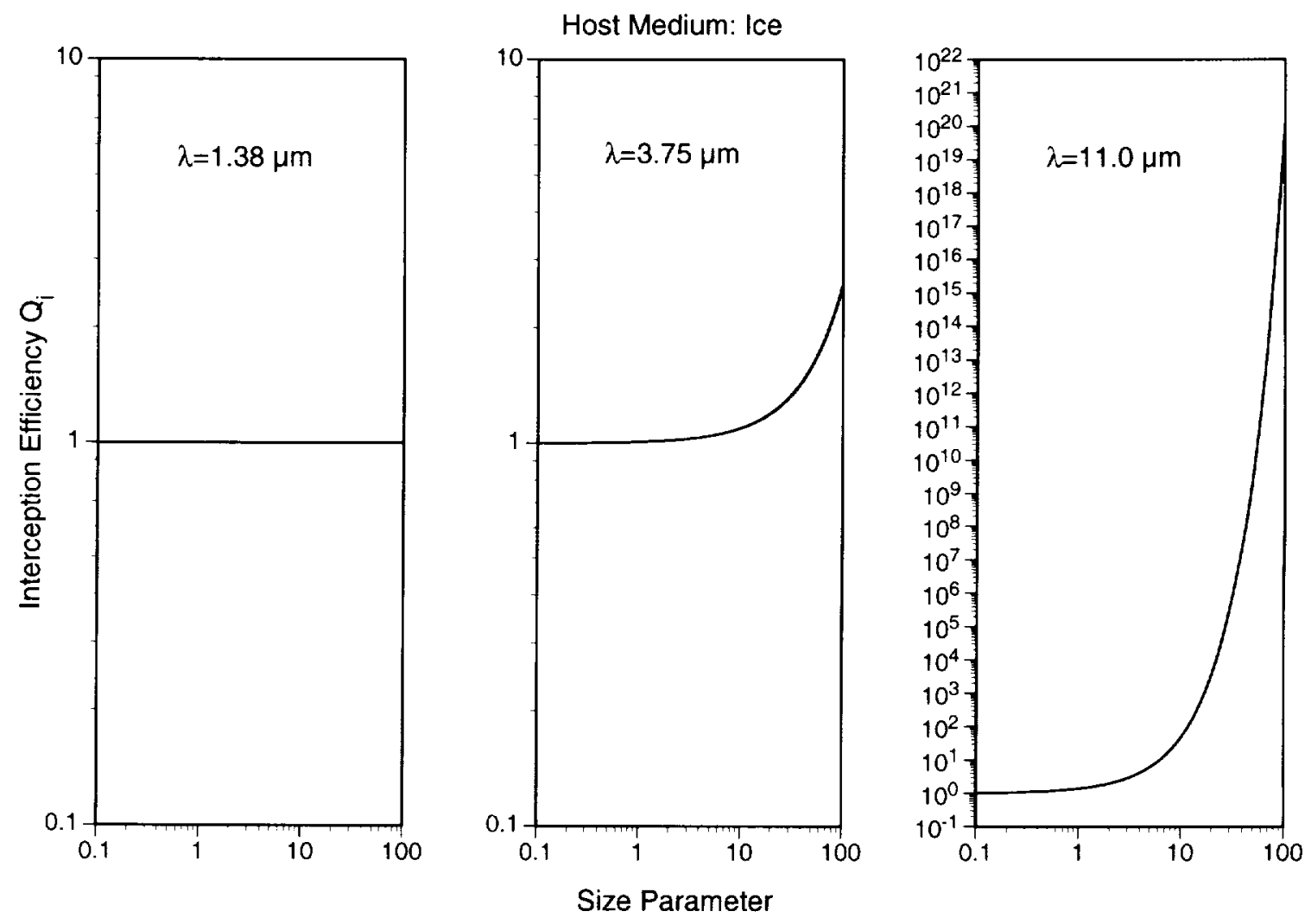

Fig.4 

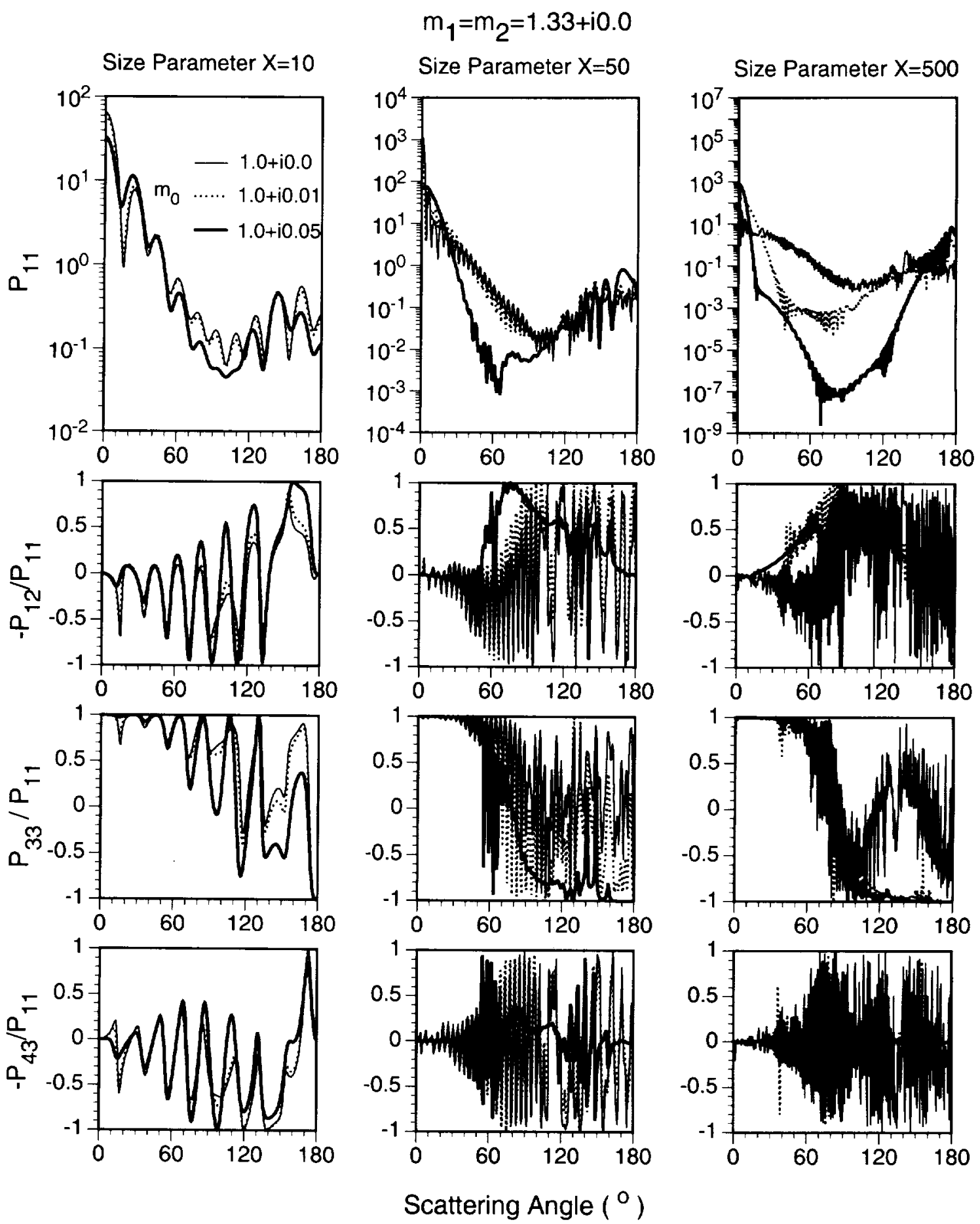

Fig.5 

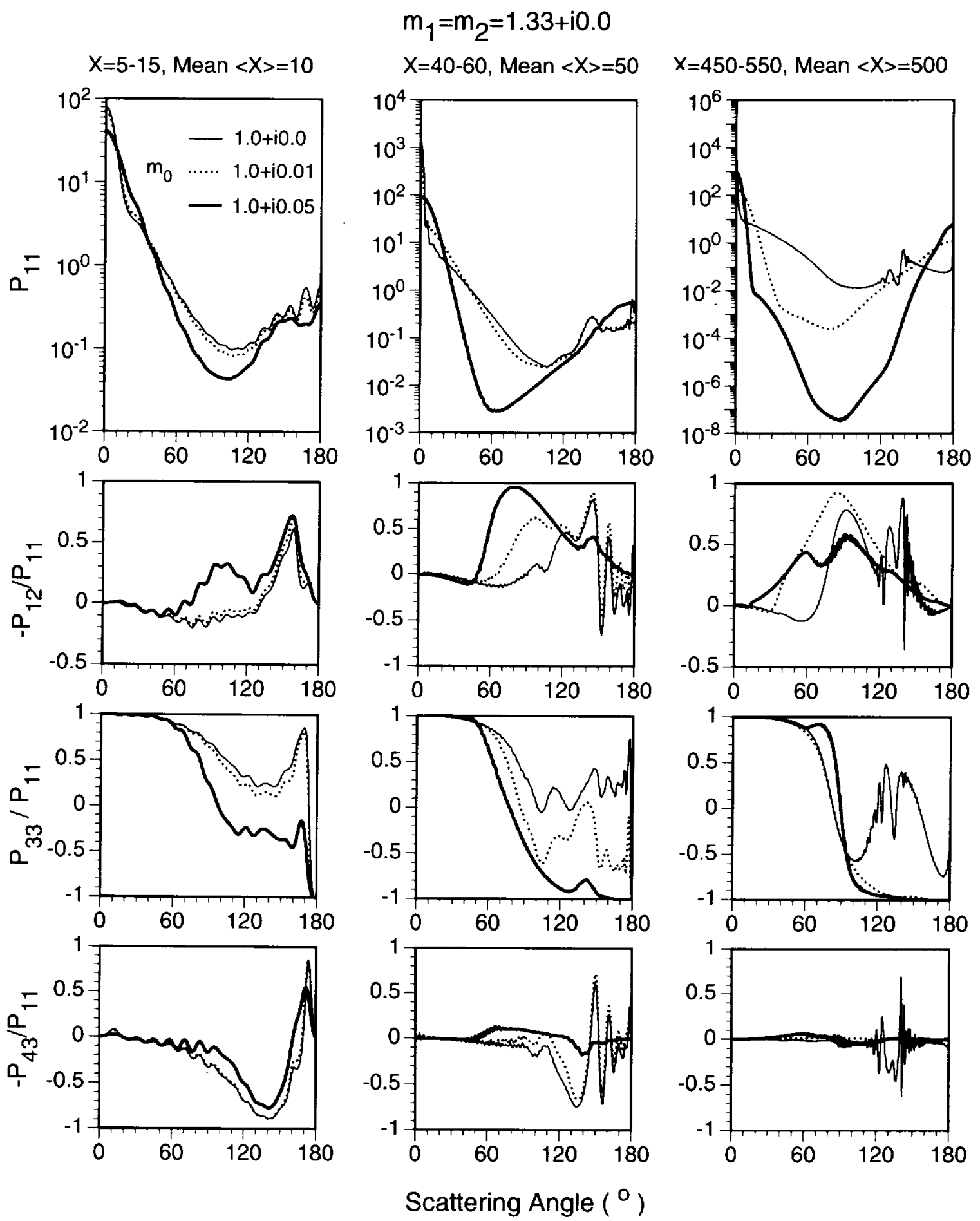

Fig.6 


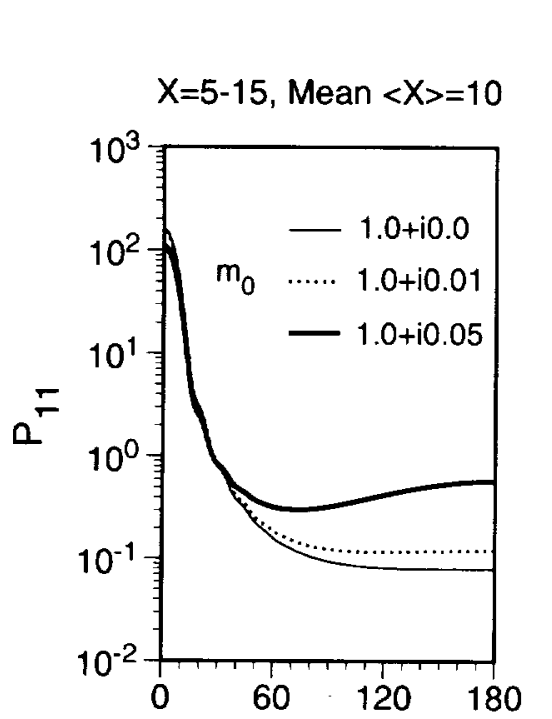

$$
\mathrm{m}_{1}=\mathrm{m}_{2}=1.75+\mathrm{i} 0.435
$$

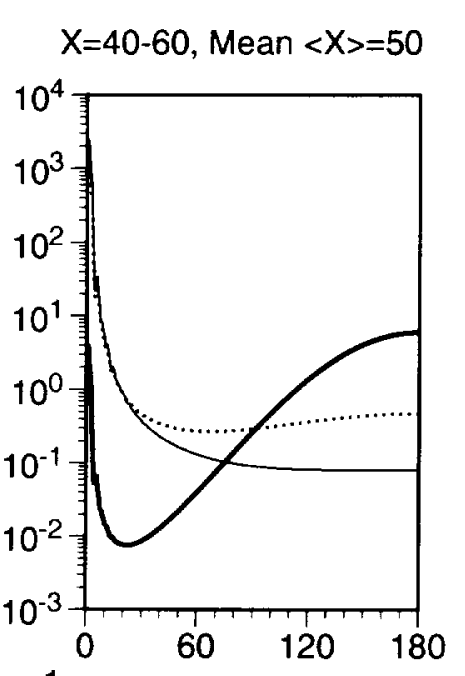

$X=450-550$, Mean $<X>=500$
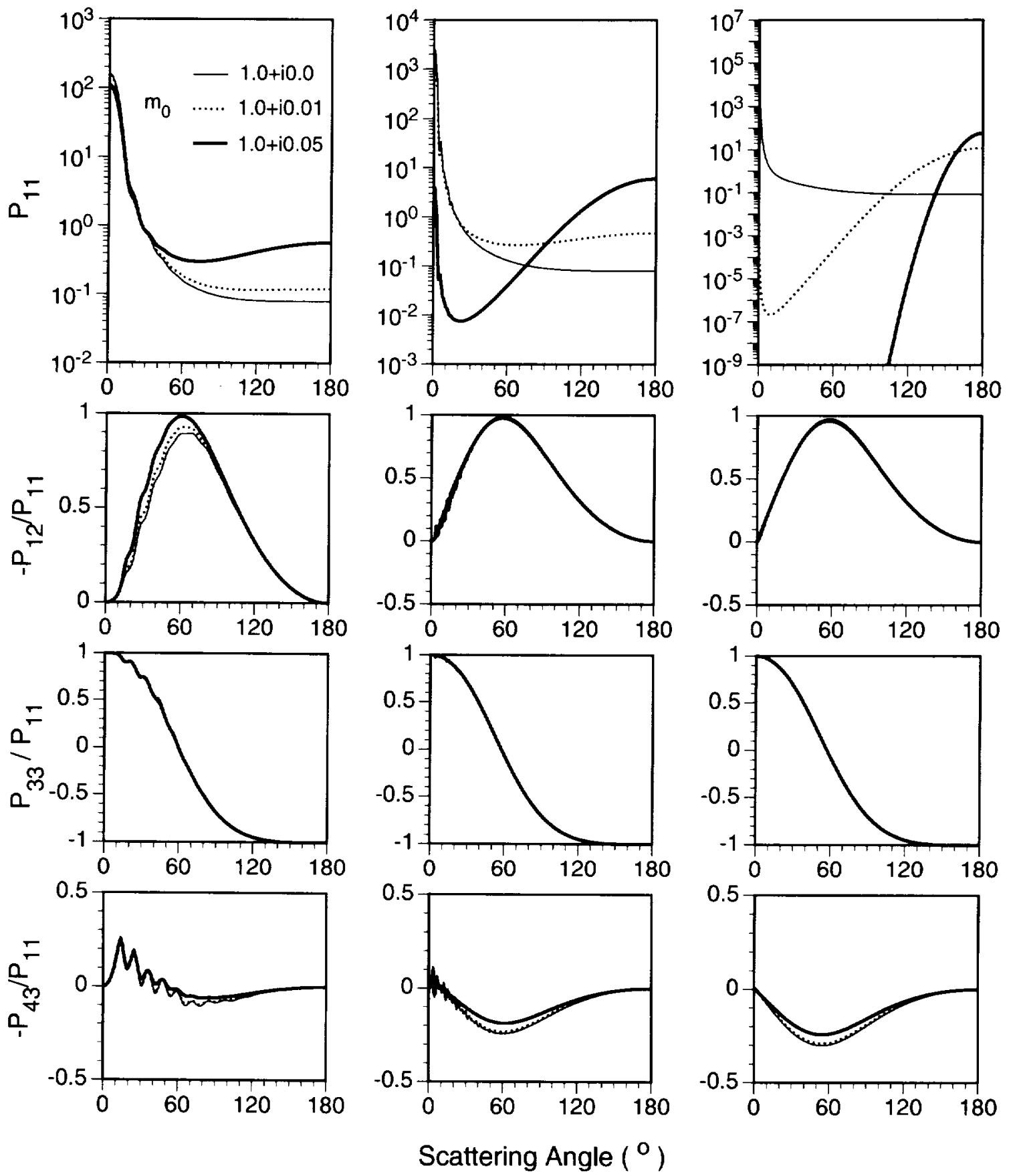

Fig.7 

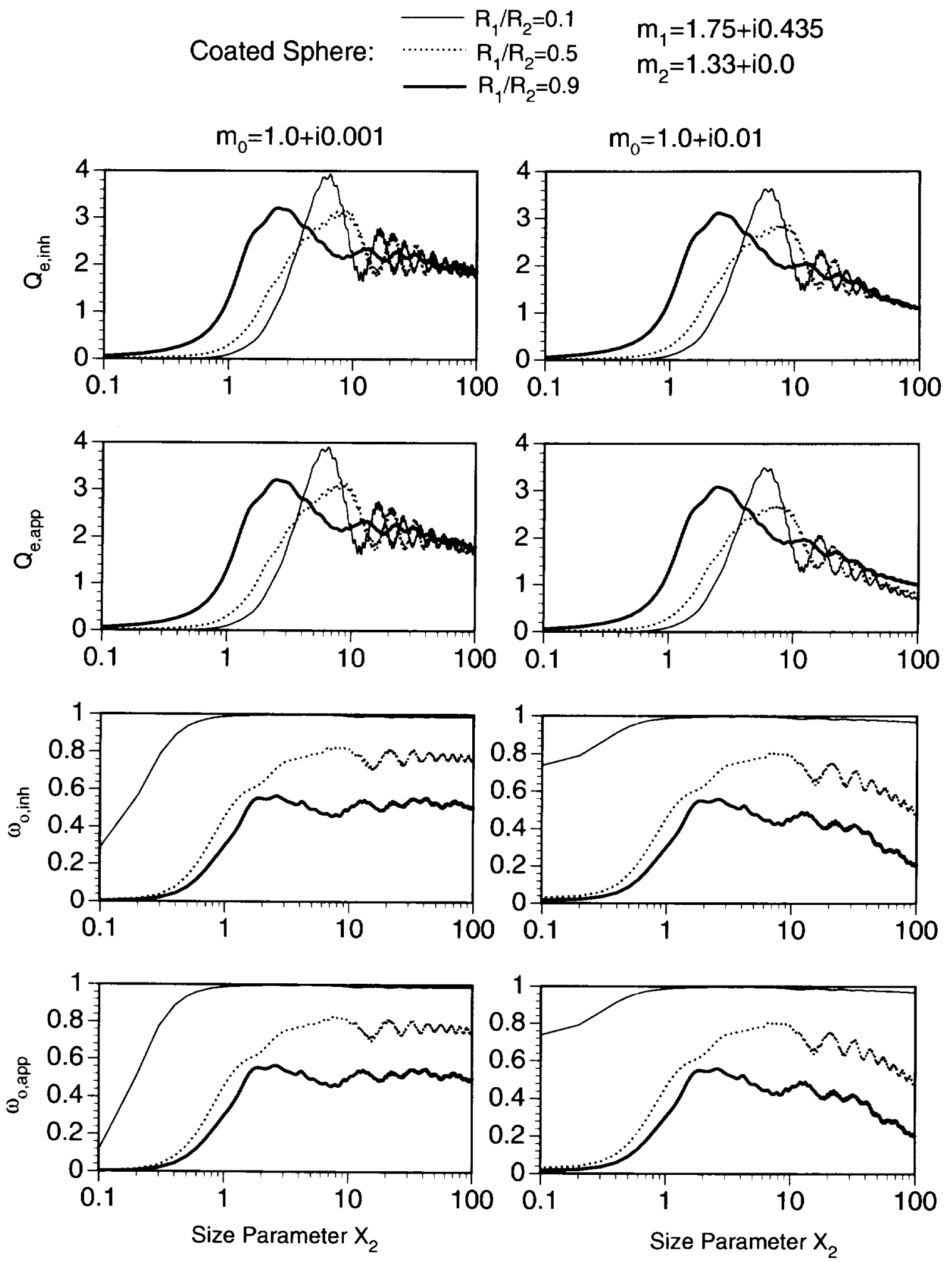

Fig.8 

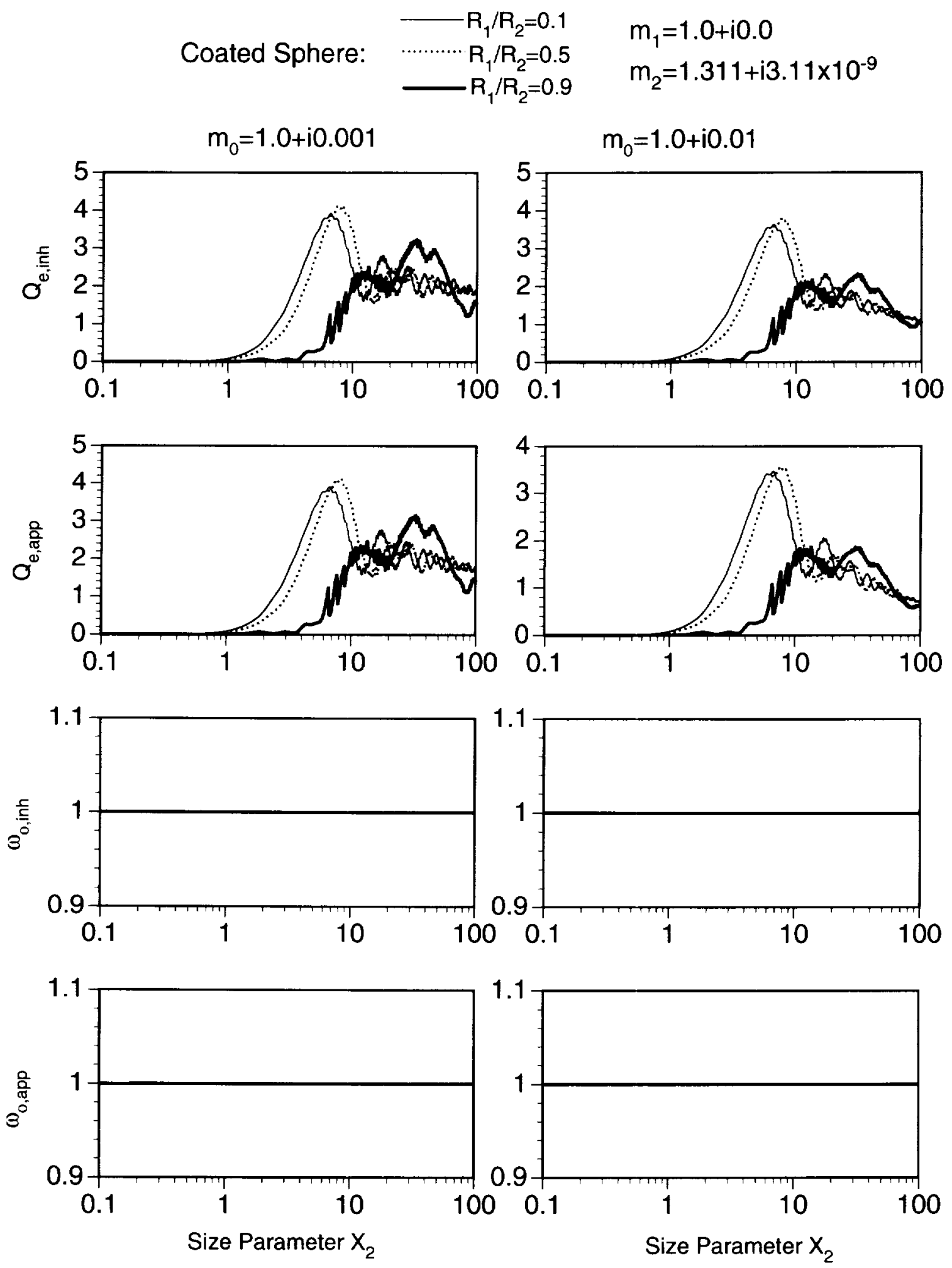

Fig.9 


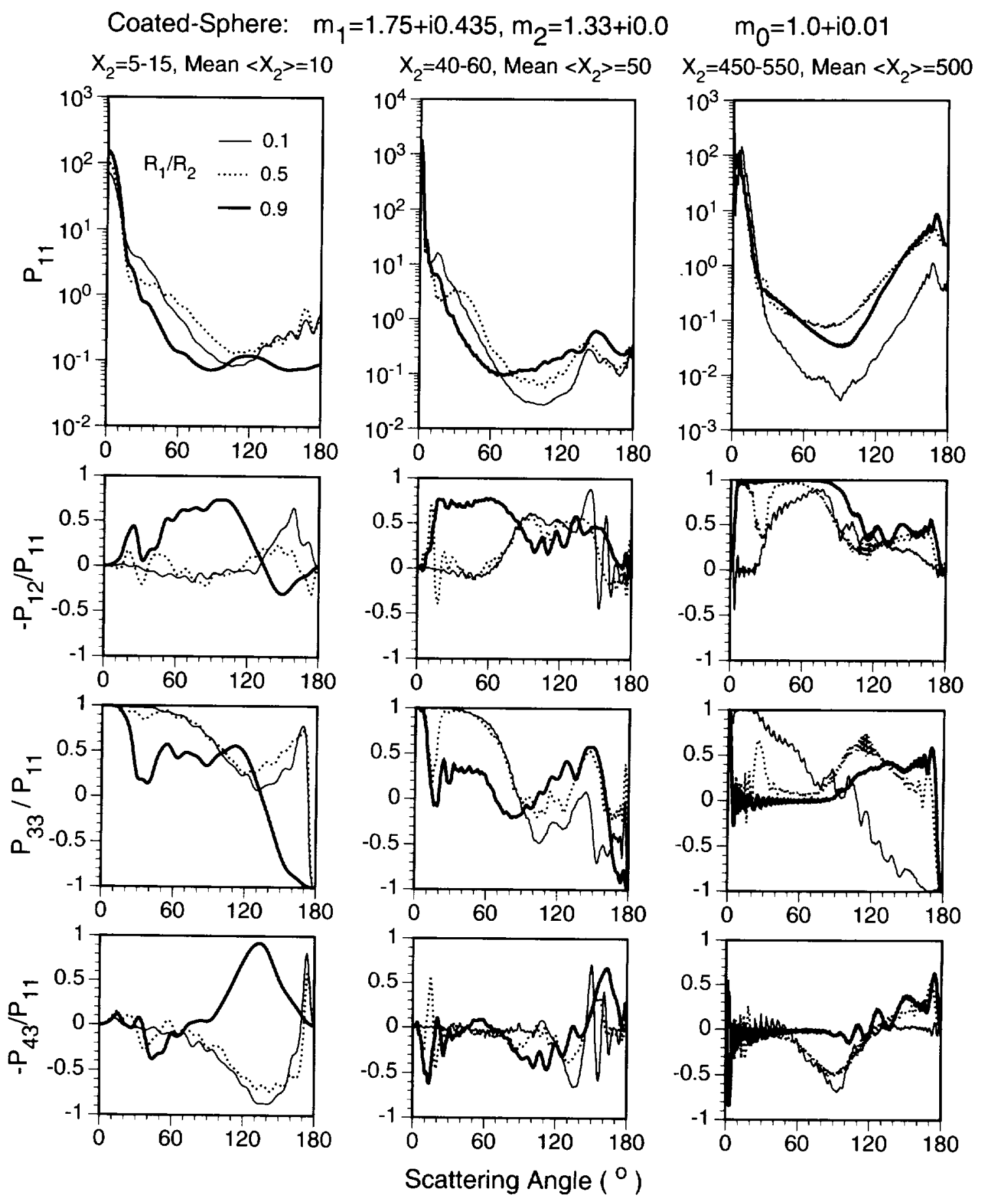

Fig.10 


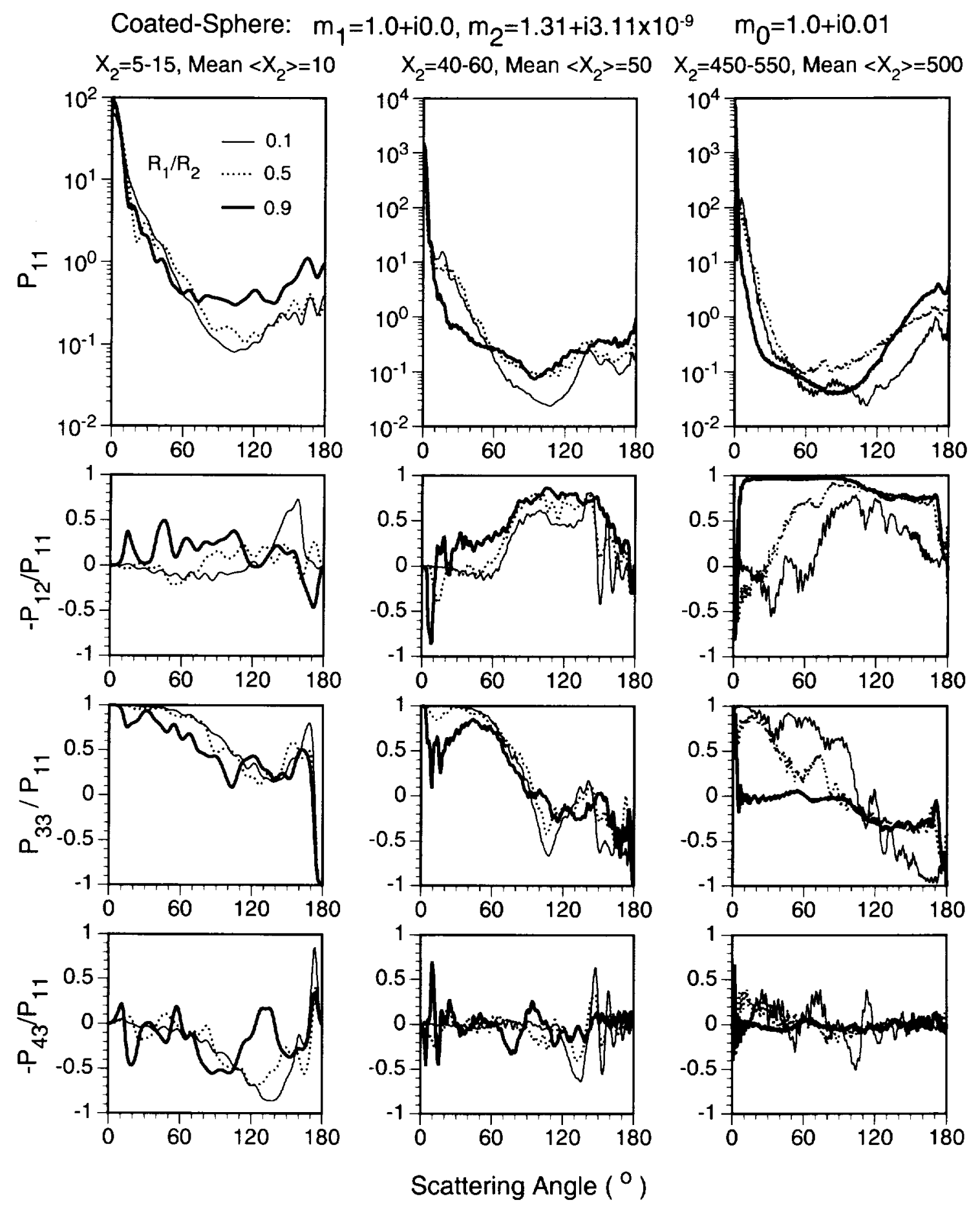

Fig.11 